\title{
Argumentstrukturmuster als Konstruktionen? Identität - Verwandtschaft - Idiosynkrasien
}

\begin{abstract}
Aus den Argumentstrukturen von Verben lassen sich vielfach eigenständige Argumentstrukturmuster mit idiosynkratischen formalen oder inhaltlichen Eigenschaften abstrahieren. Der Artikel zeigt, dass sich Ähnlichkeiten zwischen solchen Mustern nicht, wie von Goldberg (1995) vorgeschlagen, über das Konzept polysemer Argumentstrukturkonstruktionen erfassen lassen, sondern adäquater über ein Netz von Familienähnlichkeiten modelliert werden können. Die einzelnen Argumentstrukturmuster zeigen dabei eine Vielzahl von idiosynkratischen lexikalischen Kookkurrenzen, die spezifisch für die je einzelnen Argumentstrukturmuster sind und in einer implikativen Beziehung zu diesen stehen. Überlegungen zur angemessenen sprachtheoretischen Modellierung der Daten zeigen dabei sowohl Schwächen valenzbasierter Theorien als auch Mängel konstruktionsbasierter Ansätze auf. ${ }^{1}$
\end{abstract}

\section{Argumentstrukturmuster}

\subsection{Argumentstrukturmuster auf verschiedenen Abstraktionsebenen}

Die Argumentstruktur eines Lexems bringt zum Ausdruck, inwieweit seine idiosynkratischen Eigenschaften die Art der syntaktischen und semantischen Einbindung der das Lexem im Satz umgebenden Phrasen bestimmen. Unter Argumentstruktur verstehen wir dabei die einem Lexem zugeordnete Liste von Argumentstellen mit ihren syntaktischen und ihren semantischen Beschränkungen, also Beschränkungen hinsichtlich des Phrasentyps, der Kasus, der Präpositionswahl etc. einerseits und Beschränkungen hinsichtlich semantischer Rollen bzw. lexikalischer Dekomposition sowie Selektionsbeschränkungen andererseits. ${ }^{2}$ Nun lassen sich im Bereich der Argumentstrukturen von Verben auf verschiedenen Abstraktionsebenen auffällige Kovorkommen von Ausdrucksklassen beobachten, die lexemübergreifend mit bestimmten Bedeutungen einhergehen. Das legt die Vermutung nahe,

1 Die hier vorgestellten Überlegungen und Untersuchungen sind in dem Forschungsprojekt „Polysemie und konstruktionelle Varianz“ am Institut für Deutsche Sprache entstanden (www.ids-mannheim.de/lexik/PolysemieVarianz/). Für Kommentare zu dem vorliegenden Aufsatz danken wir Anke Holler.

2 Diese Auffassung von Argumentstruktur lehnt sich an ein Konzept von Valenz an, das - wie in Jacobs (1994) - syntaktische und semantische Dimensionen der Valenz unterscheidet. 
dass die Liste der semantischen und die der syntaktischen Beschränkungen lexikalischer Argumentstrukturen oft nicht in lexemspezifischer Weise aufeinander abgebildet werden, sondern wiederkehrende allgemeine Muster konstituieren wie in (1).
a. $\mathrm{X}_{\mathrm{NPnom}} \mathrm{V} \mathrm{Y}_{\mathrm{NPdat}} \mathrm{Z}_{\mathrm{NPakk}}, X$ transferiert $Z$ an $Y^{\mathrm{c}}$ z.B.: er gibt ibr das Buch/sie schickt ibm einen Brief
b. $\mathrm{X}_{\mathrm{NPnom}} \mathrm{V} \mathrm{Y}_{\mathrm{PP} \text { nach }}, X$ versucht $Y \mathrm{zu}$ finden/zu erlangen ${ }^{6}$ z.B.: sie forscht nach alten Papieren / sie jagt nach Gold
c. $\mathrm{X}_{\mathrm{NPnom}} \mathrm{VY}_{\mathrm{NPakk}} \mathrm{Z}_{\mathrm{PP} \text { nach }}, X$ versucht $Z$ in $Y$ zu finden/zu erlangen z.B. sie durchsucht die Taschen nach Geld/ sie bören den Himmel nach Signalen intelligenter Wesen $a b$
d. $\mathrm{X}_{\mathrm{NPnom}} \mathrm{V}_{\text {folgen }} \mathrm{Y}_{\mathrm{NPdat}}, X$ folgt $Y^{6}$ sie folgte dem Dieb/ sie folgen einer Spur
e. $\mathrm{X}_{\mathrm{NPnom}} \mathrm{V}_{\mathrm{V}[\text { Geräusch] }} \mathrm{Y}_{\mathrm{PP}[\text { dirt }}, X$ macht das in $V$ beschriebene Geräusch und bewegt sich entlang $Y^{6}$
z.B.: die Babn quietscht um die Ecke/ der Trecker rumpelt durch die Stadt
f. $\mathrm{X}_{\mathrm{NPnom}[\text { Motorrad] }} \mathrm{V}_{\text {knattern }} \mathrm{Y}_{\mathrm{PP} \text { durchl ibber }}$, Motorrad knattert und bewegt sich über/durch $Y^{6}$
z.B.: das Motorrad knattert durch die Stadt/ die Harleys knattern über den Highway
g. $\mathrm{X}_{\mathrm{NPnom}} \mathrm{V}_{\text {reden }} \mathrm{Y}_{\text {Refl-akk }} \mathrm{Z}_{\mathrm{PP} \text { in Rage }}, X$ redet und gerät dadurch in Rage z.B.: der Minister redet sich in Rage / du redest dich in Rage

Die konkreteren solcher Kovorkommen (z.B. $V_{\text {knattern }} \mathrm{Y}_{\mathrm{PP} \text { durrb/uiber }}$ in (1f)) lassen sich meist unmittelbar als statistisch signifikante Kookkurrenzen aus Korpora ermitteln, die kategorial bestimmten argumentstrukturbezogenen Kovorkommen (z.B. $V_{\mathrm{V}[\text { Geräusch }]} \mathrm{Y}_{\mathrm{PP}[\mathrm{dir}]}$ in (1e)) ergeben sich aus Abstraktionen über konkreten Kovorkommen. Solche argumentstrukturbezogenen, kategorial bestimmten und semantisch stabilen Kovorkommen wollen wir im Folgenden als Argumentstrukturmuster (ASM) bezeichnen.

Der Aufsatz befasst sich mit der Frage, wie sich die Identität solcher Argumentstrukturmuster bestimmen lässt und wie sie repräsentiert werden müssen. Der Umgang mit Argumentstrukturen und Argumentstrukturmustern unterscheidet sich je nach sprachtheoretischen Grundannahmen erheblich. Die meisten Grammatiktheorien sind in der einen oder anderen Weise valenz- bzw. subkategorisierungsbasiert und projizieren Argumentstrukturen aus lexikalischen Verbeinträgen. ${ }^{3}$ Die hier als Argumentstrukturmuster bezeichneten nicht-idiosynkratischen Aspekte von Argumentstrukturen werden dabei in verschiedener Weise berücksichtigt. Müller (2006) zeigt am Beispiel von Resultativkonstruktionen, wie diese Aspekte in Form lexikalischer Regeln erfasst werden können. Form-Bedeutungs-Abhängig-

In Jacobs (1992) ist schön zu sehen, wie in einem valenzgrammatischen Ansatz solche Projektionsprinzipien operieren. 
keiten in Argumentstrukturen hat man in verschiedenen Ansätzen auch über die Etablierung von Linking-Prinzipien zu erfassen versucht (z.B. Wunderlich 2006). In radikaleren Ansätzen werden aber auch andere Strategien verfolgt. So wird etwa in der Distribuierten Morphologie (z.B. Harley/Noyer 2000) ganz auf lexikalische Repräsentationen von Argumentstrukturen verzichtet, indem Argumentstrukturen, integriert in Dekompositionen, unmittelbar in der Syntax erzeugt werden. Argumentstrukturen können aber auch als eigenständige syntaktisch-semantische Entitäten betrachtet werden, so wie es die Konstruktionsgrammatik tut, die insofern für die hier Argumentstrukturmuster genannten Kookkurrenzen eigene „Argumentstrukturkonstruktionen" postuliert (Goldberg 1995, 2006). In der traditionell valenztheoretisch geprägten germanistischen Linguistik hat sich in jüngster Zeit eine Debatte entsponnen, die den Platz von Konstruktionen in valenzbasierten Grammatiken zum Gegenstand hat (Jacobs 2008, 2009; Müller 2006; Welke 2009; Handwerker 2008; Engelberg 2007, 2009).

Ziel dieses Aufsatzes ist es, ein Konzept für die Repräsentation von Argumentstrukturmustern zu entwickeln, in dem erstens fein differenzierte Argumentstrukturmuster über ein Netz von Familienähnlichkeiten verbunden sind, und zweitens idiosynkratische lexikalische Kookkurrenzen innerhalb solcher Muster repräsentiert werden können. Der Aufsatz wirft zunächst in Abschnitt 1.2 einen kritischen Blick auf die Praxis konstruktionsgrammatischer Ansätze zur Behandlung von Argumentstrukturmustern. Insbesondere werden wir Widersprüche und konzeptionelle Schwächen des Polysemiekonzepts für Argumentstrukturkonstruktionen aufdecken. Dem stellen wir in Abschnitt 2.1 Überlegungen zu einem Netz von Familienähnlichkeiten zur Repräsentation von Beziehungen zwischen Argumentstrukturmustern entgegen, die wir auf der Basis von zwei sehr unterschiedlichen Familien von Argumentstrukturmustern, den Such-Argumentstrukturmustern (Abschnitt 2.2) und den Argumentstrukturmustern mit inneren Objekten (Abschnitt 2.3) korpusbasiert unterfüttern. Darauf folgend wird zunächst am Beispiel von Bewegungslesarten von Geräuschverben (Abschnitt 3.1), dann anhand von Resultativkonstruktionen (Abschnitt 3.2) und schließlich am Beispiel von lexikalisch stark restringierten propositionalen Mustern (Abschnitt 3.3) gezeigt, dass lexikalische Kookkurrenzen innerhalb eines Argumentstrukturmusters die Repräsentation einer Vielzahl idiosynkratischer Spezifizierungen eines solchen Musters erforderlich machen.

\subsection{Die Identität von Argumentstrukturmustern}

In einer inzwischen schon als klassisch zu bezeichnenden Arbeit definiert Goldberg (1995, S. 4) Konstruktionen wie folgt: 
$\mathrm{C}$ is a CONSTRUCTION iff $_{\text {def }} \mathrm{C}$ is a form-meaning pair $\left\langle\mathrm{F}_{i}, \mathrm{~S}_{i}\right\rangle$ such that some aspect of $\mathrm{F}_{i}$ or some aspect of $\mathrm{S}_{i}$ is not strictly predictable from $\mathrm{C}^{\prime}$ s component parts or from other previously established constructions. ${ }^{4}$

Dabei macht die Konstruktionsgrammatik bekanntermaßen keinen Unterschied zwischen atomaren (nicht komplexen) und komplexen Konstruktionen. Wörter und syntaktische Regelhaftigkeiten konstituieren also gleichermaßen Konstruktionen und werden in einem einheitlichen Format repräsentiert (Goldberg 1995, S. 7). Allgemeine Eigenschaften, die das Inventar atomarer Konstruktionen - also etwa Wörter - strukturieren, sollten daher auch im Inventar komplexer Konstruktionen - also etwa Argumentstrukturkonstruktionen - wirken. Und genau das wird in Goldberg (ebd.) auch angenommen. Zu den inventarstrukturierenden Eigenschaften, die wir aus dem Lexikon kennen, gehören Homonymie und Polysemie. Im Falle von Polysemie werden mehrere Form-Bedeutungseinheiten, deren Formen identisch und deren Bedeutungen verwandt sind, einem Zeichen zugeordnet. Im Falle von Homonymie werden dagegen Form-Bedeutungseinheiten, deren Formen identisch und deren Bedeutungen nicht miteinander verwandt sind, verschiedenen Zeichen zugewiesen. Goldberg beobachtet, dass Polysemie im Lexikon sehr häufig, Homonymie dagegen selten auftritt. Eine solche Verteilung sollte daher auch im Bereich komplexer Konstruktionen zu erwarten sein (Goldberg 2009). Goldberg (1995, S. 33 f.) illustriert dies am Beispiel von Argumentstrukturkonstruktionen, von denen sie annimmt, dass sie typischerweise polysem sind. Sie konstituieren damit ein Zeichen mit mehreren Bedeutungen. ${ }^{5}$ So hat etwa die Ditransitiv-Konstruktion neben der zentralen Transferbedeutung, die durch Verben wie give exemplifiziert wird, eine Reihe weiterer Bedeutungen, u.a. die durch die folgenden Beispiele repräsentierten:

(2) a. Chris baked Jan a cake.

b. Bill promised his son a car.

c. Joe allowed Bill a popsicle.

d. Joe refused Bill a raise in salary.

Jede dieser Lesarten der Ditransitivkonstruktion weist nach Goldberg eine semantische Idiosynkrasie auf und ist auf die zentrale Lesart bezogen. ${ }^{6}$ In (2a) ist der durch die zentrale Lesart gegebene Transfer nicht impliziert, sondern lediglich intendiert, in (2b) ist er in die Glückensbedingungen des

4 In Goldberg (2006, S. 5) wird der Konstruktionsbegriff dahingehend erweitert, dass nicht nur formale oder semantische Idiosynkrasien, sondern auch Gebrauchshäufigkeiten die Existenz von Konstruktionen begründen können.

5 Goldberg spricht von „senses“, wobei wir „sense“ hier im Sinne von „Lesart“ als eine von mehreren miteinander verwandten Bedeutungen eines Zeichens verstehen.

6 Die zentrale Lesart wird dadurch identifiziert, dass sie konkret ist, diachron primär und Grundlage für metaphorische Erweiterungen (Goldberg 1995, S. 33). 
Verbs eingebunden, in (2c) wird er lediglich ermöglicht, und in (2d) negiert (Goldberg 1997, S. 392). Aufgrund der Polysemieannahme sind alle Sätze in (2) Exemplifikationen der gleichen Konstruktion. Gegenüber dem häufigen Auftreten von Polysemie bei Argumentstrukturkonstruktionen sei Homonymie dagegen, so Goldberg (2006, S. 36), bei Argumentstrukturkonstruktionen sehr selten. Als eines der seltenen Beispiele führt sie forAdverbial-Konstruktionen an, die mit den beiden nicht aufeinander bezogenen Bedeutungen in (3) verknüpft sind. Hier haben wir es nach Goldberg also mit zwei verschiedenen Konstruktionen zu tun.

a. The statue stood for three hours.

b. He exchanged the socks for a belt.

Die Annahme konstruktioneller Polysemie beinhaltet allerdings sowohl theorieinterne Widersprüche als auch konzeptionelle Schwächen. Das zentrale Argument Goldbergs (1995, S. 34) für die Zurückführung von Beispielen wie (2a) auf eine eigene Bedeutungsvariante $\left[F, B_{2}\right]$ der Ditransitivkonstruktion basiert darauf, dass die spezifischen Eigenschaften dieser Variante nicht semantisch regelhaft ableitbar oder pragmatisch zu erschließen, sondern bis zu einem gewissen Maße unprädiktabel seien. Die Nicht-Prädiktabilität eines Aspekts eines Form-Bedeutungspaares wie $\left[F, B_{2}\right]$ ist aber gemäß der einleitenden Definition von Konstruktionen das zentrale Kriterium für das Vorliegen einer eigenständigen Konstruktion. Das ist ein offensichtlicher Widerspruch. Will man also Polysemie von Konstruktionen zulassen, muss man auch die Definition von Konstruktionen revidieren und wie folgt rekonstruieren:

\section{Konstruktionsidentität unter der Annahme konstruktioneller Polysemie}

$K$ ist eine Konstruktion $\operatorname{gdw}_{\text {def }}$ gilt: $K$ ist ein Paar $\left[F,<B_{1}, \ldots, B_{n}>\right]$ aus genau einer Form $F$ und einer nicht leeren Liste von Bedeutungen $<B_{1}, \ldots, B_{n}>$, wobei

(i) mindestens ein Aspekt von $F$ oder mindestens ein Aspekt von jedem $B$ aus $<B_{1}, \ldots, B_{n}>$ nicht strikt aufgrund der konstituierenden Teile von $K$ oder aufgrund anderer, bereits etablierter Konstruktionen prädiktabel ist, und

(ii) jede Bedeutung $B$ aus $<B_{1}, \ldots, B_{n}>$ semantisch auf die zentrale Bedeutung $B_{1}$ bezogen ist.

Kein Paar $[F, B]$ für ein beliebiges $B$ aus $<B_{1}, \ldots, B_{n}>$ ist eine Konstruktion.

Der letzte Punkt ergibt sich daraus, dass ansonsten zwei Paare, $\left[F, B_{2}\right]$ und $\left[F, B_{3}\right]$, eigenständige Konstruktionen wären und aufgrund ihrer Formidentität damit homonym. Und genau das wird ja explizit von Goldberg negiert. 
Schaut man sich nun den weiteren Umgang Goldbergs mit konstruktioneller Polysemie an, so muss man feststellen, dass die von ihr als Lesarten bestimmten Varianten von Konstruktionen tatsächlich oft wie eigenständige Konstruktionen behandelt werden. Das zeigt sich vor allem im Umgang mit den für Konstruktionsgrammatiken üblichen Vererbungsprinzipien, die es erlauben, geteilte Informationen verschiedener Konstruktionen zentral zu repräsentieren und von dort quasi zu verteilen. Vererbung wird dabei aufgefasst als eine Relation zwischen Konstruktionen (Goldberg 1995, S. 73). Bezüglich der Lesarten von Argumentstrukturkonstruktionen wird nun so verfahren, dass die Bedeutung der einzelnen Lesarten mit der zentralen Lesart über so genannte „polysemy links“ verknüpft ist, die die jeweils spezifische Art der semantischen Beziehung beschreiben. Die syntaktischen Eigenschaften der einzelnen Lesarten werden dagegen von der zentralen Lesart vererbt (Goldberg 1995, S. 75). Dass die Lesarten (semantische „extensions") nun plötzlich wie eigenständige Konstruktionen behandelt werden, macht Goldberg dann auch explizit:

Each of the extensions constitutes a minimally different construction, motivated by the central sense; that is, each sense can be represented by a construction that is minimally different from that of the central sense. (Goldberg 1995, S. 76)

Damit werden Form-Bedeutungs-Paare wie $\left[F, B_{2}\right]$ und $\left[F, B_{3}\right]$ nun gleichzeitig als Varianten eines einzelnen polysemen konstruktionellen Zeichens behandelt wie auch als zwei homonyme konstruktionelle Zeichen. Neben diesem Widerspruch hat Goldbergs Auffassung von konstruktioneller Polysemie unseres Erachtens aber auch eine konzeptionelle Schwäche. Diese resultiert aus der Überstrapazierung der Parallelen zwischen atomaren und komplexen Konstruktionen. Goldbergs von uns rekonstruierte Auffassung von Konstruktionsidentität unter der Annahme konstruktioneller Polysemie fordert strikte Formidentität der Form-Bedeutungspaare, aber keine strikte Bedeutungsidentität. Die Ditransitivkonstruktion konstituiert also in ihren semantischen Varianten genau ein abstraktes Zeichen und nicht mehrere. Dagegen führt Bedeutungsidentität bei divergierenden Formen nicht zu Konstruktionsidentität, sondern zur Annahme von verschiedenen synonymen bzw. semantisch eng verwandten Konstruktionen. ${ }^{7}$ Man mag sich fragen, warum das so ist. Schauen wir uns Fälle von Synonymie bzw. enger Bedeutungsverwandtschaft im Lexikon an, so stellen wir fest, dass synonyme Zeichen wie Orange und Apfelsine gewöhnlich in ihrer Form stark divergieren. Das motiviert bei aller Bedeutungsgleichheit oder - in anderen Fällen - enger Bedeutungsverwandtschaft die Annahme, dass es sich um unterschiedliche Zeichen handelt. Nun ist im Bereich von Argumentstruk-

Goldberg (1995, S. 67) nimmt ein „Principle of No Synonymy“ an, demzufolge es keine echte Synonymie gibt. Das im Folgenden angeführte Argument gilt aber auch, wenn verschiedene Formen nicht die gleiche, aber doch eine sehr ähnliche Bedeutung haben. 
turkonstruktionen allerdings manchmal festzustellen, dass synonyme bzw. semantisch eng verwandte Konstruktionen anders als im Fall von Orange und Apfelsine formal nur partiell differieren.

a. Sie untersuchten die Daten nach Schlüsselwörtern.

b. Sie untersuchten die Daten auf Schlüsselwörter.

Die beiden zugrundeliegenden Argumentstrukturmuster unterscheiden sich nur an einer Stelle, nämlich in der Wahl der Präposition: ${ }^{8}$
a. $\mathrm{X}_{\mathrm{NPnom}} \mathrm{V} \mathrm{Y}_{\mathrm{NPakk}} Z_{\mathrm{PP} \text { nacb }}, X$ versucht $Z$ in $Y$ zu erlangen
b. $\mathrm{X}_{\mathrm{NPnom}} \mathrm{V} \mathrm{Y}_{\mathrm{NPakk}} Z_{\mathrm{PP} \text { auf }}, X$ versucht $Z$ in $Y$ zu erlangen ${ }^{6}$

Da die beiden Muster auch in der Bedeutung kaum differieren, ${ }^{9}$ stellt sich hier unter der Annahme, dass Konstruktionen prinzipiell mehrere Varianten in sich vereinigen können, die Frage, warum ein Goldbergscher Ansatz es nicht zulässt, (5a) und (5b) als formale Varianten einer Konstruktion aufzufassen.

Wir haben gesehen, dass Goldbergs Zeichenkonzept in Bezug auf Argumentstrukturkonstruktionen durch Widersprüche gekennzeichnet ist. Ein und dieselbe partiell idiosynkratische Form-Bedeutungseinheit wird einmal als Konstruktion in homonymer Relation zu anderen Konstruktionen aufgefasst, einmal als Lesart einer Konstruktion in Relation zu anderen Lesarten. Zudem erscheint die Polysemieannahme auch dahingehend ad hoc, dass Form-Bedeutungseinheiten mit gleichen Formen und ähnlichen Bedeutungen einer Konstruktion zugeordnet werden, solche mit gleichen Bedeutungen und ähnlichen Formen aber verschiedenen Konstruktionen. Beides lässt das Polysemiekonzept von Konstruktionen fragwürdig erscheinen.

Wir wollen daher im Folgenden auf der Basis empirischer Korpusstudien zeigen, dass Varianten von Argumentstrukturmustern sich eher als ein Netz von Familienähnlichkeiten zwischen granular modulierten einzelnen Argumentstrukturmustern konstituieren denn als ein hierarchisierendes Polysemiekonzept.

\section{Familienähnlichkeiten bei Argumentstrukturmustern}

\subsection{Familienähnlichkeiten statt konstruktioneller Polysemie}

Angelehnt an Wittgensteins (2001) Konzept von Familienähnlichkeiten konzipieren wir Strukturen innerhalb der Menge von Argumentstruk-

Vgl. die Belege in Proost (2009).

Es sei dahingestellt, ob die beiden Varianten synonym sind oder geringfügige Bedeutungsdifferenzen aufweisen. Sie stehen sich semantisch aber sicherlich näher als die Varianten der Ditransitivkonstruktion. 
turmustern als Familien. Dabei gehen wir davon aus, dass eine Familie sich als ein Netz von Familienmitgliedern konstituiert, die anderen, aber nicht allen Mitgliedern der Familie hinreichend ähnlich sein müssen.

\section{Familienähnlichkeit bei Argumentstrukturmustern}

Für $\mathrm{R}$ als eine Relation hinreichender Ähnlichkeit bezüglich der Werte einer Menge von Attributen $\{\alpha, \beta, \gamma, \ldots\}$ und $\mathrm{A}$ als eine Menge von $n$ Argumentstrukturmustern gilt:

$A$ ist in Bezug auf $R$ eine Familie, $\mathfrak{F}^{A R}$, gdw. es für jede Teilmenge $A^{T} \subset A$ und ihre Komplementmenge ${ }^{10} \mathrm{~A}^{\mathrm{K}} \subset \mathrm{A}$ mindestens ein Paar von Argumentstrukturmustern, $a^{t} \in A^{T}$ und $a^{k} \in A^{K}$, gibt, die in einer Relation hinreichender Ähnlichkeit, $\mathrm{R}\left(\mathrm{a}^{\mathrm{t}}, \mathrm{a}^{\mathrm{k}}\right)$, stehen.

Die Ähnlichkeitsrelation $\mathrm{R}$ wird in Bezug auf bestimmte Attribute definiert, in unserem Fall also etwa die Anzahl der Argumente in einem Muster, die formalen Eigenschaften der Argumentstellen in den einzelnen Mustern, die semantischen Eigenschaften zur Charakterisierung der Muster etc. Wann zwei Argumentstrukturmuster in Bezug auf die Werte dieser Attribute als hinreichend ähnlich gelten, lässt diese Definition offen. Das ist gesondert zu operationalisieren.

Die formulierte Bedingung für Familienähnlichkeit stellt zum einen sicher, dass jedes Argumentstrukturmuster mindestens einem anderen Muster der Familie ähnlich ist. Das ergibt sich daraus, dass (i) jedes einzelne Argumentstrukturmuster eine Teilmenge konstituiert und dieses dann zu einem Muster in der Komplementmenge in Beziehung steht und (ii) in jeder Teilmenge, die mehrere einander ähnliche Argumentstrukturmuster enthält, wiederum mindestens ein Argumentstrukturmuster eine Ähnlichkeitsbeziehung ins Komplement dieser Teilmenge unterhält. Die Bedingung stellt damit sicher, dass alle Argumentstrukturmuster in das Ähnlichkeitsnetz eingebunden sind, das die Familie konstituiert. Wäre lediglich verlangt, dass jedes Muster in A zu mindestens einem anderen Muster in A in einer Ähnlichkeitsbeziehung steht, so wäre diese Bedingung in einer Menge von 4 Mustern schon durch $\mathrm{R}\left(\mathrm{a}^{1}, \mathrm{a}^{2}\right)$ und $\mathrm{R}\left(\mathrm{a}^{3}, \mathrm{a}^{4}\right)$ erfüllt. Das ist zur Konstituierung einer Familie aber sicherlich nicht hinreichend, denn $\mathrm{a}^{1}$ und $\mathrm{a}^{2}$ einerseits und $\mathrm{a}^{3}$ und $\mathrm{a}^{4}$ andererseits sind nun ihrerseits nicht mehr miteinander verbunden. Der Umweg über die Partitionierung von A in sämtliche mögliche Teilmengen und ihre Komplemente verhindert das.

Die Relation $\mathrm{R}$ ist hier als binäres kategoriales Merkmal \pm HINREICHEND_ÄHNLICH konzipiert. Als solches ist sie reflexiv, da jedes Argumentstrukturmuster sich natürlich selbst hinreichend ähnlich ist, und sym-

${ }_{10} \quad \mathrm{~A}^{\mathrm{T}}$ und $\mathrm{A}^{\mathrm{K}}$ sind also disjunkt, d.h. ihre Schnittmenge ist leer, und sie schöpfen A aus, d.h. ihre Vereinigungsmenge ist gleich A. 
metrisch, da dann, wenn ein Argumentstrukturmuster $\mathrm{a}^{1}$ einem Muster $\mathrm{a}^{2}$ ähnlich ist, umgekehrt $\mathrm{a}^{2}$ auch $\mathrm{a}^{1}$ ähnlich ist. $\mathrm{R}$ ist aber nicht transitiv. ${ }^{11} \mathrm{Das}$ heißt, wenn $\mathrm{a}^{1}$ und $\mathrm{a}^{2}$ hinreichend ähnlich sind und $\mathrm{a}^{2}$ und $\mathrm{a}^{3}$ ebenfalls, so folgt daraus nicht, dass auch $\mathrm{a}^{1}$ und $\mathrm{a}^{3}$ hinreichend ähnlich sind. Das ergibt sich daraus, dass die Ähnlichkeit zwischen $\mathrm{a}^{1}$ und $\mathrm{a}^{2}$ auf anderen Attributen basieren kann als die zwischen $\mathrm{a}^{2}$ und $\mathrm{a}^{3}$. So ist (6a) (6b) semantisch hinreichend ähnlich, und (6b) ist (6c) formal hinreichend ähnlich. Daraus folgt aber nicht, dass (6a) und (6c) einander hinreichend ähnlich sind.
a. $\mathrm{X}_{\mathrm{NPakk}} \mathrm{V}, X$ macht die in $V$ ausgedrückte psychische Erfahrung' (z.B. ibn friert)
b. $\mathrm{X}_{\mathrm{NPnom}} \mathrm{V}, X$ macht die in $V$ ausgedrückte psychische Erfahrung (z.B. er friert)
c. $\mathrm{X}_{\mathrm{NPnom}} \mathrm{V}, X$ führt die in $V$ ausgedrückte psychische Handlung aus $^{6}$
(z.B. er denk.t nach)

Die Nicht-Transitivität der Ähnlichkeitsbeziehung ist unserer Auffassung nach ein wichtiger Bestandteil eines Wittgensteinschen Konzepts von Familienähnlichkeiten, das nur sehr abstrakte notwendige Bedingungen für die Familienzugehörigkeit verlangt - in dem hier vorliegenden Fall lediglich, dass alle Familienmitglieder Argumentstrukturmuster sind. Die Nicht-Transitivität der Ähnlichkeitsbeziehung beinhaltet, dass es darüber hinaus keine weiteren Eigenschaften gibt, die notwendigerweise von allen Familienmitgliedern geteilt werden.

Das vorgestellte Konzept von hinreichender Ähnlichkeit entspricht als kategoriales Konzept natürlich nicht einem intuitiven Konzept von Ähnlichkeit als gradueller Eigenschaft. Ein Familienähnlichkeitskonzept für Argumentstrukturmuster könnte natürlich auch auf einer ordinalen Konzeption von Ähnlichkeit beruhen, in der auf der Basis der Anzahl der Übereinstimmungen in den Attributwerten zu $\{\alpha, \beta, \gamma, \ldots\}$ Stufen von Ähnlichkeit unterschieden werden. Diese Abstufung könnte gegebenenfalls durch die unterschiedliche Gewichtung einzelner Merkmale ergänzt werden.

Über die Menge der Ähnlichkeitsrelationen zu anderen Mitgliedern der Familie und gegebenenfalls über die Stärke dieser Ähnlichkeitsrelationen können schließlich zentrale Muster innerhalb einer Familie von Argumentstrukturmustern ermittelt werden. Letztlich lässt das vorgestellte Konzept erwarten, dass sich alle Argumentstrukturmuster in einer großen Familie wiederfinden werden. Rund um die zentralen Muster werden sich eng miteinander verwandte Argumentstrukturmuster zu clusterähnlichen Teilfamilien gruppieren.

$11 \quad$ Reflexivität: $\mathrm{R}\left(\mathrm{a}^{1}, \mathrm{a}^{1}\right)$. Symmetrie: $\mathrm{R}\left(\mathrm{a}^{1}, \mathrm{a}^{2}\right) \Rightarrow \mathrm{R}\left(\mathrm{a}^{2}, \mathrm{a}^{1}\right)$. Transitivität: $\mathrm{R}\left(\mathrm{a}^{1}, \mathrm{a}^{2}\right) \& \mathrm{R}\left(\mathrm{a}^{2}, \mathrm{a}^{3}\right) \Rightarrow$ $\mathrm{R}\left(\mathrm{a}^{1}, \mathrm{a}^{3}\right)$. 
Ein Beispiel für eine über Familienähnlichkeiten strukturierte Menge von Argumentstrukturmustern findet sich im Anhang. Dort werden die Ähnlichkeiten innerhalb der Familie der Such-Argumentstrukturmuster, die im folgenden Abschnitt 2.2 ausschnittsweise näher beschrieben wird, tabellarisch und als Abbildung dargestellt.

Es sei hier darauf hingewiesen, dass auch Goldberg den Terminus ,Familienähnlichkeit` für das Beziehungsgefüge von Argumentstrukturkonstruktionen für sich beansprucht:

[...] we have treated resultatives as a sort of ,family' of constructions (we might call them subconstructions of the resultative), sharing important properties but differing in certain specifics, including their degree of productivity. By, family' we have in mind the sort of family resemblances recognized to exist in non-linguistic categories (Rosch \& Mervis 1975, Wittgenstein 1955). Our strategy here is first to establish a taxonomy of the subconstructions, then to state which of their properties can be explained by the construction as a whole, and finally pick out some of the differences. (Goldberg/Jackendoff 2004, S. 535 f.)

Ein Unterschied zu unserem Konzept ergibt sich hier daraus, dass Goldberg und Jackendoff auf der einen Seite von Familienähnlichkeiten im Sinne Wittgensteins sprechen, auf der anderen Seite jedoch (i) die Notwendigkeit bestimmter, sehr eng gefasster, ,important properties“ konstatieren, die allen Familienmitgliedern gemein sein müssen, und (ii) zudem die Konstruktionen in einer „taxonomy of the subconstructions“ organisieren. Insbesondere Letzteres entspricht allerdings gerade nicht dem Begriff der Familienähnlichkeit bei Wittgenstein, der auf der Annahme beruht, dass die Mitglieder einer Familie eben nicht notwendig offensichtliche zentrale Eigenschaften miteinander teilen, sondern in feineren Netzen der oben beschriebenen Art miteinander verknüpft sind. Gerade der Schwierigkeit der taxonomischen Erfassung und Hierarchisierung von Begriffen ist Wittgensteins Ansatz der Familienähnlichkeit geschuldet, der sich eben solcher Mengen von Ausdrücken annimmt, die unklare Grenzen aufweisen. Das im vorliegenden Aufsatz beschriebene Konzept von Familienähnlichkeit unterscheidet sich jedenfalls in dieser Hinsicht von dem Konzept, das Goldberg/Jackendoff (2004) entwickeln.

In den folgenden beiden Abschnitten werden wir zwei sehr unterschiedliche Familien von Argumentstrukturmustern auf der Basis von Korpusdaten näher betrachten. Sie sollen das Konzept einer granularen Auffassung von Argumentstrukturmustern illustrieren, die in ihren formalen und inhaltlichen Ähnlichkeiten Familien im oben beschriebenen Sinn konstituieren. ${ }^{12}$

12 Die Daten entstammen alle dem unter COSMAS II zugänglichen Deutschen Referenzkorpus am Institut für Deutsche Sprache (www.ids-mannheim.de/kl/projekte/korpora/). 


\subsection{Die Familie der Such-Argumentstrukturmuster}

Such-Argumentstrukturmuster sind Argumentstrukturmuster, mit denen ausgedrückt wird, dass der Subjektreferent den Referenten der in der PP eingebetteten NP zu finden, zu erlangen oder zu ermitteln versucht (vgl. Die Touristen suchen nach ibren Reisedokumenten. Der Jurist forscht nach Motiven für die Tat.). Insgesamt lassen sich vier Typen von Such-Argumentstrukturmustern unterscheiden: (i) Such-Argumentstrukturmuster mit Bezug auf nicht-mentale, nicht-sprachliche Handlungen, (ii) Such-Argumentstrukturmuster mit Bezug auf mentale Handlungen, (iii) Such-Argumentstrukturmuster mit Bezug auf sprachliche Handlungen und (iv) Such-Argumentstrukturmuster mit Bezug auf Einstellungen. Da jedem dieser vier Typen von Such-Argumentstrukturmustern verschiedene Varianten zugeordnet werden können, entsprechen den Typen (i)-(iv) vier Subfamilien der Familie der Such-Argumentstrukturmuster. Jedes Such-Argumentstrukturmuster kann insofern als separates Argumentstrukturmuster betrachtet werden, als es der Definition für Argumentstrukturmuster genügt:

\section{Argumentstrukturmuster}

Eine Kombination von überzufällig stark kookkurrierenden sprachlichen Einheiten ist genau dann ein Argumentstrukturmuster, wenn (i) diese Einheiten aus einem verbalen Element und seinen Argumenten bestehen, (ii) mindestens eine dieser Einheiten nicht durch lexikalische Elemente, sondern durch kategoriale semantische und/oder syntaktische Spezifizierungen gekennzeichnet ist, und (iii) mindestens ein formaler oder semantischer Aspekt dieser Spezifizierung nicht aus allgemeinen Festlegungen der Grammatik (Syntax/Morphologie/Semantik/Pragmatik) oder des Lexikons hergeleitet werden kann.

Die Nicht-Prädiktabilität von Aspekten eines Argumentstrukturmusters kann dabei die Realisierungsform und Anzahl der Argumente, die Bedeutung des Musters oder die Klassen von Verben betreffen, die in ihm auftreten. Die Definition erlaubt die Unterscheidung abstrakter von konkreten Argumentstrukturmustern durch den Grad der Generalität der kategorialen Beschreibung.

Im Folgenden wird am Beispiel einiger ausgewählter Such-Argumentstrukturmuster gezeigt, dass diese bestimmte Idiosynkrasien aufweisen, die dafür sprechen, sie als separate Argumentstrukturmuster anzusehen. Argumentstrukturmuster werden dabei als Paare bestehend aus einer Form F und einer Bedeutung $\mathrm{B}$ dargestellt, so dass beispielsweise das Muster ASM1 als eine Verbindung einer Form1, $\mathrm{F}_{1}$, und einer Bedeutung1, $\mathrm{B}_{1}$, ASM2 als eine Verbindung von $\mathrm{F}_{2}$ und $\mathrm{B}_{2}$ usw. repräsentiert wird. Insgesamt lassen sich 13 Such-Argumentstrukturmuster unterscheiden. In diesem Beitrag werden nur 8 von diesen diskutiert; die übrigen sind in der Übersichtsgra- 
fik im Anhang aufgeführt. (Die Nummerierung der im Text diskutierten Such-Argumentstrukturmuster bezieht sich auf die Nummerierung in dieser Übersichtsgrafik.)

\subsubsection{Such-Argumentstrukturmuster mit Bezug auf nicht-mentale, nicht-sprachliche Handlungen}

Das semantisch zentrale Muster der Familie der Such-Argumentstrukturmuster stellt das Muster ,nach etwas suchen' (ASM1) dar. Es stellt semantisch den Ausgangspunkt für die Konstitution der Familie dar und nimmt Verben (wie z.B. suchen) auf, die der Argumentstrukturmusterbedeutung sehr nahe stehen. Es steht nicht in einer übergeordneten taxonomischen Relation zu den anderen Argumentstrukturmustern der Familie. ASM1 besteht in formaler Hinsicht aus einem Verb, einer NP im Nominativ und einer PP mit nach und hat die Bedeutung , $X$ versucht, $Y$ durch Ausführen der Handlung $V$ zu finden, zu erlangen, zu ermitteln', vgl.:

ASM1: nach etwas suchen

F: $\mathrm{X}_{\mathrm{NPnom}} \mathrm{V} \mathrm{Y}_{\mathrm{PP} \text { nacb }}$

B: $X$ versucht, $Y$ durch Ausführen der Handlung $V$ zu finden, zu erlangen, zu ermitteln.

Das Muster ASM1 wird durch den folgenden Beleg aus den IDS-Korpora exemplifiziert:

(7) [Die Verlage, die überleben wollen $]_{\mathrm{X}}$, [suchen $]_{\mathrm{V}}$ händeringend [nach literarischer Ware] $]_{Y}$ (Der Spiegel, 17.01.1994; ERZÄHLER MÜSSEN HER [S. 147])

ASM1 ist in semantischer Hinsicht zunächst insofern unprädiktabel, als es nur mit Verben bestimmter semantischer Klassen vorkommt (Such-Verben, Verben wie graben, Bewegungsverben, Verben wie kratzen, Wahrnehmungsverben und Verben des Anstrengens und Bemühens) und nicht beispielsweise mit Herstellungsverben wie bauen oder mit Verben, mit denen auf mentale Aktivitäten Bezug genommen wird, wie etwa lesen. Denkbare Äußerungen wie z.B. Er baut nach einem neuen Haus oder Sie liest nach einer Lösung kommen in den Korpora nicht vor. Dass durch die Verbindung des ASM1 mit Verben der oben erwähnten Klassen die Interpretation ,suchen bzw. ,auf eine bestimmte Art und Weise suchen' evoziert wird (vgl. nach neuen Klamotten schauen bzw. nach Zeichen von Verschütteten lauschen), ist ebenfalls unprädiktabel. Ähnlich argumentiert Goldberg mit Bezug auf die Ditransitiv-Konstruktion, dass es keinen erkennbaren Grund dafür gibt, warum diese Konstruktion nur mit Verben bestimmter semantischer Klassen vor- 
kommt und warum durch ihre Verbindung mit Verben bestimmter semantischer Klassen eine ganz bestimmte Interpretation entsteht (vgl. Goldberg 1995, S. 34). Über die bereits erwähnten Gründe hinaus ist ASM1 auch deswegen unprädiktabel, weil es innerhalb der Klassen von Verben, die dieses Muster prinzipiell erlauben, immer auch eine beträchtliche Anzahl von Verben gibt, die in den Korpora nicht mit ASM1 vorkommen (z.B. ergründen und erwieren in der Klasse der Such-Verben, gaffen und kosten in der Klasse der Wahrnehmungsverben, wetzen und eilen in der Klasse der Bewegungsverben usw. (vgl. Proost 2009, S. 17 f.).

Das Argumentstrukturmuster ASM2 (,auf etwas angeln) unterscheidet sich nur darin von ASM1, dass das Argument der Entität, die der Subjektreferent zu erlangen versucht, nicht als PP mit nach, sondern als PP mit auf realisiert wird. Dementsprechend kann dieses Muster folgendermaßen dargestellt werden: ${ }^{13}$

ASM2: auf etwas angeln

$\mathrm{F}: \mathrm{X}_{\mathrm{NPnom}} \mathrm{V} \mathrm{Y}_{\mathrm{PP} \text { auf }}$

B: $X$ versucht, $Y$ durch Ausführen der Handlung $V$ zu erlangen.

ASM2 wird durch Belege wie (8) illustriert:

(8) Eigentlich [hat $]_{\mathrm{V}}[\text { Frank Pauli }]_{\mathrm{X}}[\text { auf Aal }]_{\mathrm{Y}}[\text { geangelt }]_{\mathrm{V}}$, stand im Mondlicht geduldig am Neckar-Schifffahrtskanal und wartete auf einen dicken Fisch, der wollte jedoch nicht anbeißen. (Mannheimer Morgen, 01.07.2004; Mondschein-Geschichte mit Haaren und Haken)

Da ASM2 sich in formaler Hinsicht von ASM1 unterscheidet und im Gegensatz zu diesem nur mit einigen wenigen Verben (angeln, fischen, jagen und gehen) vorkommt, stellt es ein eigenes Argumentstrukturmuster dar. Unprädiktabel ist vor allem, warum die anderen Such-Verben, die mit ASM1 auftreten, nicht als Teil von ASM2 vorkommen.

Eine dritte Variante der Such-Argumentstrukturmuster, das Muster ASM3 (etwas nach etwas durchsuchen'), unterscheidet sich sowohl formal als auch im Hinblick auf seine Bedeutung von den beiden bereits erwähnten Such-Argumentstrukturmustern. Zusätzlich zu den beiden Argumenten, die auch ASM1 und ASM2 enthalten (derjenige, der sucht, und die gesuchte Entität) enthält das Muster ASM3 das Argument der Entität, in der gesucht wird. Dementsprechend hat ASM3 die Bedeutung, $X$ versucht, $Y$ durch Ausführen der Handlung $V$ in $Z$ zu finden, zu erlangen, zu ermitteln'. ASM3 kann folgendermaßen als ein Form-Bedeutungspaar dargestellt werden:

13 Es ist außerdem zu beobachten, dass das in die PP eingebettete Substantiv in manchen Fällen im Singular artikellos steht. 
ASM3: etwas nach etwas durchsuchen

$\mathrm{F}: \mathrm{X}_{\mathrm{NPnom}} \mathrm{V} \mathrm{Z}_{\mathrm{NPakk}} \mathrm{Y}_{\mathrm{PP} \text { nach }}$

B: $X$ versucht, $Y$ durch Ausführen der Handlung $V$ in $Z$ zu finden, zu erlangen, zu ermitteln.

ASM3 wird durch Belege wie (9) illustriert:

(9) Am Sonntag brachen unbekannte Täter ebenfalls tagsüber in eine Erdgeschoßwohnung in der Welserstraße ein. [Sie $]_{\mathrm{X}}[\text { durchsuchten }]_{\mathrm{V}}$ [alles $]_{Z}[\text { nach Wertgegenständen }]_{Y}$. (Mannheimer Morgen, 07.05.1996, Lokales; Hundehalterin nach Unfallflucht gesucht)

Obwohl man die Bedeutung von ASM3 als eine Erweiterung der Bedeutung von ASM1 (,nach etwas suchen) ansehen könnte, ist die Tatsache, dass dieses Argumentstrukturmuster ein zusätzliches Argument in einer bestimmten semantischen Rolle enthält und sich damit formal von ASM1 und ASM2 unterscheidet, ein Grund, es als ein eigenständiges Argumentstrukturmuster zu betrachten.

Das Argumentstrukturmuster ASM4 (,etwas auf etwas durchsuchen') unterscheidet sich nur darin von ASM3 (,etwas nach etwas durchsuchen'), dass das Argument der gesuchten Entität nicht als PP mit nach, sondern als PP mit auf realisiert wird, vgl.:

ASM4: etwas auf etwas durchsuchen

F: $\mathrm{X}_{\mathrm{NPnom}} \mathrm{V} \mathrm{Z}_{\mathrm{NPakk}} \mathrm{Y}_{\mathrm{PP} \text { auf }}$

B: $X$ versucht, $Y$ durch Ausführen der Handlung $V$ in $Z$ zu finden, zu erlangen, zu ermitteln.

ASM4 ist durch das folgende Beispiel belegt:

(10) Wer dennoch eine Impfung scheut, $[\mathrm{der}]_{\mathrm{X}}[\text { sollte }]_{\mathrm{V}}$ wenigstens geschlossene Kleidung tragen und [seine Sachen $]_{Z}$ zuhause nach der Tour [auf Zecken] $]_{\mathrm{Y}}$ [durchsuchen] $]_{\mathrm{V}}$. (Frankfurter Rundschau, 04.06. 1999, S. 36, Ressort: HESSEN; Zwei Krankheiten und ihre Verbreitungsgebiete)

Das Argumentstrukturmuster ist insofern unprädiktabel, als es nicht mit allen Verben vorkommt, die als Teil von ASM3 auftreten können. Durchwühlen kann beispielweise als Teil von ASM3, aber nicht als Teil von ASM4 vorkommen. 


\subsubsection{Such-Argumentstrukturmuster mit Bezug auf mentale Handlungen}

Während die Argumentstrukturmuster ASM1-ASM4 ausdrücken, dass der Subjektreferent den Referenten der in der PP eingebetteten NP zu finden, zu erlangen oder zu ermitteln versucht, indem er eine bestimmte Handlung ausführt, drücken die Muster ASM6 (,nach etwas sinnen') und ASM7 (,auf Rache/Revanche/Vergeltung sinnen') aus, dass der Subjektreferent den Referenten der in der PP eingebetteten NP durch Ausführen einer mentalen Handlung zu ermitteln versucht. Das Argumentstrukturmuster ASM6, das durch Beispiel (11) illustriert ist, kann folgendermaßen dargestellt werden:

ASM6: nach etwas sinnen

F: $X_{\text {NPnom }} V Y_{\text {PPnach }}$

B: $X$ versucht, $Y$ durch Ausführen der mentalen Handlung $V$ zu ermitteln.

(11) Olaf Nicolai freute sich, dass seine raumfüllende „Flamme der Revolution, liegend (in Wolfsburg)" noch einmal aufgestellt wurde. Ohne Sponsoren und das Engagement der Städtischen Galerie wäre das nicht möglich gewesen, und deshalb $[\mathrm{sann}]_{\mathrm{V}}[\mathrm{er}]_{\mathrm{X}}$ gestern schon [nach Ideen, wie er der Galerie etwas Gutes tun könnte] $]_{Y}$. Braunschweiger Zeitung, 02.03.2007; Zum fröhlichen Künstler-Bahnhof im Schloss)

ASM6 ist insofern idiosynkratisch, als es zwar mit Verben des Denkens (brïten, grübeln, rätseln, sich den Kopf / das Hirn zermartern, sinnen und sinnieren), nicht aber mit denken und überlegen vorkommt. Dass durch die Verbindung der Verben dieser Klasse mit dem Muster ASM6 die Interpretation ,durch Überlegen suchen' hervorgerufen wird, ist ebenfalls nicht vorhersagbar.

ASM7 (,auf Rache/Revanche/Vergeltung sinnen') (vgl. Beleg (12)) ist eine idiomatische Spezialisierung von ASM6 (,nach etwas sinnen?). In formaler Hinsicht unterscheidet es sich darin von Letzterem, dass das Argument der gesuchten Entität als PP mit auf realisiert wird und die Menge der in der PP eingebetteten nominalen Ausdrücke auf einige wenige Nomina wie Rache, Revanche und Vergeltung beschränkt ist. Dementsprechend ist die Bedeutung von ASM7 spezifischer als die von ASM6. Das Argumentstrukturmuster ASM7 kann als die folgende Verbindung von Form und Bedeutung dargestellt werden: 
ASM7: auf Rache/Revanche/Vergeltung sinnen

$\mathrm{F}: \mathrm{X}_{\mathrm{NPnom}} \mathrm{V} \mathrm{Y}_{\mathrm{PP} \text { auf }}$

B: $X$ versucht, $Y$ (eine Möglichkeit der Rache) durch Ausführen der mentalen Handlung $V$ zu ermitteln.

(12) Das Sozialzentrum habe ihm die Wohnung gekündigt und ihn nicht weiter beschäftigen wollen. [Der psychisch labile Mann $]_{\mathrm{X}}[\text { habe }]_{\mathrm{V}}$ deshalb [auf Rache] $]_{\mathrm{Y}}$ [gesonnen] $]_{\mathrm{V}}$. (Berliner Zeitung, 31.08.2004; ExMitarbeiter steckte jüdisches Zentrum an [S. 6])

Obwohl die Bedeutung von ASM7 als eine Spezialisierung der Bedeutung von ASM6 betrachtet werden kann, hat ASM7 aufgrund seiner formalen Idiosynkrasien den Status eines eigenständigen Argumentstrukturmusters.

\subsubsection{Such-Argumentstrukturmuster mit Bezug auf sprachliche Handlungen}

Im Gegensatz zu den Mustern ASM6 und ASM7, die Bezug auf mentale Handlungen haben, drückt das Muster ASM9 (,nach etwas schreien') aus, dass der Subjektreferent den Referenten der in der PP eingebetteten NP für sich oder für eine dritte Person zu erlangen versucht, indem er einen impliziten Adressaten dazu auffordert, etwas zu tun, um das Gewünschte zu beschaffen. Form und Bedeutung von ASM9 können folgendermaßen dargestellt werden:

ASM9: nach etwas schreien

F: $\mathrm{X}_{\mathrm{NPnom}} \mathrm{V} \mathrm{Y}_{\mathrm{PP} \text { nach }}$

B: $X$ versucht, $Y$ zu erlangen, indem er einen impliziten Adressaten mit der sprachlichen Handlung $V$ dazu auffordert, etwas zu tun, damit $X$ oder eine 3. Person $Y$ bekommt.

ASM9 wird durch Belege wie (13) instanziiert:

(13) Wirtschafts- und Finanzbehörde lehnen ein solches staatliches Sponsoring aus grundsätzlichen Erwägungen ab, der Senat mag ebenfalls nicht einsehen, daß [ein privatwirtschaftliches Unternehmen] $]_{\mathrm{X}}$ immer dann [nach staatlicher Hilfe $]_{\mathrm{Y}}\left[\right.$ schreit $_{\mathrm{V}}$, wenn's ökonomisch nicht so prächtig funktioniert hat. (die tageszeitung, 13.04.1991, S. 28; Kein Herz für den Hamburger SV)

Das Vorhandensein eines impliziten Adressaten als Komponente der Bedeutung von ASM9 stellt eine semantische Idiosynkrasie dar, die dafür spricht, ASM9 als ein eigenes Argumentstrukturmuster innerhalb der Familie der Such-Argumentstrukturmuster zu betrachten. 


\subsubsection{Such-Argumentstrukturmuster mit Bezug auf Einstellungen}

Schließlich könnten auch Argumentstrukturmuster wie ASM13 (auf etwas hoffen) (vgl. Beleg (14)) zur Familie der Such-Argumentstrukturmuster gerechnet werden. Im Gegensatz zu allen bereits diskutierten Mustern haben solche wie ASM13 Bezug auf Einstellungen. Obwohl ASM13 den Mustern ASM1-ASM10 formal sehr ähnlich ist, unterscheidet es sich im Hinblick auf seine Bedeutung deutlich von ihnen. Semantisch ist ASM13 den bereits diskutierten Mustern nur insofern ähnlich, als der Subjektreferent den Referenten der in der PP eingebetteten NP antizipiert, ohne selber dabei handelnd in Erscheinung zu treten. ASM13 kann als die folgende Verbindung von Form und Bedeutung dargestellt werden:

ASM13: auf etwas hoffen

F: $\mathrm{X}_{\mathrm{NPnom}} \mathrm{V} \mathrm{Y}_{\mathrm{PPauf}}$

B: $X$ antizipiert $Y$ in der durch $V$ ausgedrückten Weise.

(14) „Was ist das gemeinsame von Gasometer und Aktentasche?" [Der Ausbildungsplatzanwärter, der diese Frage beim Intelligenz-Struktur-Test im Rahmen seiner Bewerbung richtig beantwortet $]_{\mathrm{X}}$, [kann $]_{\mathrm{V}}$ [auf Beschäftigung $]_{\mathrm{Y}}$ [hoffen $]_{\mathrm{V}}$. (die tageszeitung, 24.10.1986, S. 9; Bildungspolitische Notizen)

ASM13 kommt nur mit einigen wenigen Verben (boffen und warten) vor.

Die beobachteten Idiosynkrasien der Muster ASM1-ASM13 haben gezeigt, dass diese nach der Definition der Identität von Argumentstrukturmustern separate Argumentstrukturmuster sind. Da keine der Bedeutungen $B_{1}-B_{13}$ als zentrale Lesart angesehen werden kann, auf die alle anderen Bedeutungen bezogen werden könnten, können die Muster ASM1ASM13 nicht als Varianten einer einzigen polysemen Konstruktion betrachtet werden. Stattdessen strukturieren sich die Muster in Form von Familienähnlichkeiten, so wie es in der Tabelle und den Grafiken im Anhang dargestellt ist. Tabelle 1 zeigt, welche semantischen und formalen Ähnlichkeiten zwischen Such-ASM1-13 bestehen. Die Ähnlichkeiten wurden jeweils für ein Paar von Such-ASM (ASMx-ASMy, vgl. Spalte 1) aufgeführt. Als semantische Ähnlichkeiten wurden das Vorkommen beider ASM in der gleichen Subfamilie sowie ihre Übereinstimmung im Hinblick auf die Anzahl ihrer Argumente (beide 2 Argumente vs. beide 3 Argumente) berücksichtigt (Spalten 2-4). Als formale Ähnlichkeiten zählten die Übereinstimmung der beiden ASM hinsichtlich ihrer Präposition (beide nach, beide auf oder beide um) sowie die Übereinstimung der in ihnen vorkommenden phrasalen Kategorien (d.h. ob beide eine NPnom und/oder beide eine NPakk enthalten) (vgl. Spalten 5-9). Übereinstimmung zwischen zwei ASM in Bezug auf 
eines oder mehrere dieser Merkmale wurde mit „,““ markiert. Aus der Anzahl der übereinstimmenden Merkmale wurde das Maß der Ähnlichkeit zwischen den ASM eines Paares errechnet, wobei alle Merkmale als gleichwertig angesehen wurden. Insgesamt variierte der Grad der Ähnlichkeit der ASM zwischen 1 und 4, d.h. es gibt ein bis vier gemeinsame Merkmale (vgl. letzte Spalte). In Abbildung 13 wird der Grad der Ähnlichkeit von Such-ASM exemplarisch für ASM1, ASM3, ASM4 und ASM9 dargestellt. Der Grad der semantischen und formalen Ähnlichkeit zwischen diesen ASM wird durch die unterschiedliche Linienstärke repräsentiert.

\subsection{Die Familie der Argumentstrukturmuster mit inneren Objekten}

Die Gemeinsamkeiten und Unterschiede zwischen den Varianten von Argumentstrukturmustern, die innere Objekte enthalten, lassen sich ebenfalls über Familienähnlichkeiten beschreiben. Es gibt sowohl Varianten, die die gleiche Form, aber eine andere Bedeutung haben, als auch Varianten, die bedeutungsgleich oder bedeutungsähnlich sind, und eine unterschiedliche Form haben. Alle diese Argumentstrukturmuster bestehen aus einem verbalen Argument und seinen Elementen, wobei meistens mehrere dieser Einheiten durch kategoriale semantische und /oder syntaktische Spezifizierungen gekennzeichnet sind, und auch mindestens ein formaler oder semantischer Aspekt dieser Spezifizierungen nicht prädiktabel ist aufgrund von allgemeinen Festlegungen der Grammatik oder des Lexikons. So stellt das innere Objekt in den meisten Fällen eine Argumenterweiterung bei normalerweise intransitiv verwendeten Verben dar, die nicht aus allgemeinen Festlegungen herleitbar ist. Auch die morphologische und etymologische Verwandtschaft des Objektnomens mit dem Verb, die häufig vorhanden ist, ist nicht auf diese Weise prädiktabel. Außerdem sind alle hier besprochenen Varianten von Argumentstrukturmustern, die innere Objekte enthalten, insofern idiosynkratisch, als in keiner Weise vorhersagbar ist, mit welchen Verben sie vorkommen können oder nicht (vgl. zu den einzelnen Varianten Winkler 2009).

Das Muster ASM14 ist das wohl häufigste und wird im Allgemeinen sowohl unter formalen als auch unter semantischen Gesichtspunkten mit inneren Objekten assoziiert (vgl. u.a. Baron 1971; Matsumoto 1996; Höche 2009).

ASM14: ein Lächeln lächeln

F: $\mathrm{X}_{\mathrm{NPnom}} \mathrm{V} \mathrm{Y}_{\mathrm{NPakk}}$

B: $X$ führt eine Handlung $V$ aus, und $V$ ist mit dem von $Y$ Bezeichneten ereignis-identisch. 
ASM14 wird durch Belege wie (15) illustriert:

(15) An die Adresse der Rebellen gerichtet sagte Rice: „Sie sollten wissen, dass $[\text { wir }]_{\mathrm{X}}$ [diesen Kampf $]_{\mathrm{Y}}[\mathrm{kämpfen} \mathrm{werden}]_{\mathrm{V}}$, bis er siegreich beendet ist." Die Sicherheitslage in Afghanistan hat sich in den vergangenen Monaten dramatisch verschlechtert. (dpa, 28.06.2006; (Zusammenfassung 1545 - neu: Panzerfaustbeschuss statt Sprengsatz) Wieder Anschlag auf Bundeswehr in Kundus - drei Soldaten verletzt)

Das Objektsnomen in ASM14 ist morphologisch, etymologisch und / oder semantisch mit dem Verb verwandt und wird in den meisten Fällen durch Modifikation noch weiter spezifiziert. Diese Form des inneren Objekts kommt in der Mehrzahl der Fälle bei intransitiven Verben vor und stellt dann eine Argumenterweiterung dar. ASM14 kommt bei Verben wie gehen, hassen, kämpfen, lachen, lächeln, laufen, leben, schlafen, spielen, sterben, tanzen oder tun vor.

Das Muster ASM15 ist ebenso wie das folgende ASM16 auf das Verb sterben festgelegt, was auch in der Formkomponente der Beschreibung bereits angegeben ist. Bis auf diese Festlegung ist ASM15 formgleich mit ASM14; das Objektsnomen ist aber nur semantisch mit dem Verb verwandt und wird stets durch Modifikation weiter spezifiziert.

ASM15: einen qualvollen Tod sterben

F: $\mathrm{X}_{\mathrm{NPnom}} \mathrm{V}_{[\text {sterben] }} \mathrm{Y}_{\mathrm{NPakk}}$

B: $X$ befindet sich in dem Prozess $V$, und $Y$ ist das Resultat von $V$.

ASM15 ist durch einen Beleg wie (16) illustriert:

(16) [Mehr als 5.000 Menschen, überwiegend Frauen und Kinder $]_{\mathrm{X}}$, [starben $]_{V}$ in der irakischen Stadt [einen qualvollen Tod $]_{Y}$. Die Produktion des Giftgases wurde im Irak erst durch Lieferungen deutscher Firmen ermöglicht. (die tageszeitung, 17.03.1989, S. 2; Giftgasopfer - schon vergessen?)

Die Variante ASM16 von Argumentstrukturmustern mit innerem Objekt ist mit ASM15 bedeutungsgleich und unterscheidet sich durch einen einzelnen Formaspekt von diesem: Das innere Objekt wird hier in einem anderen Kasus realisiert als in ASM15, nämlich im Genitiv statt im Akkusativ:

ASM16: eines natürlichen Todes sterben

$\mathrm{F}: \mathrm{X}_{\mathrm{NPnom}} \mathrm{V}_{[\text {sterben }]} \mathrm{Y}_{\mathrm{NPgen}}$

B: $X$ befindet sich in dem Prozess $V$, und $Y$ ist das Resultat von $V$. 
ASM16 wird durch Beispiele wie (17) belegt:

(17) Vor kurzem warteten Wissenschaftler aus Bozen mit einer weiteren dramatischen Neuigkeit auf: [«Ötzi» $]_{\mathrm{X}}[\text { starb }]_{\mathrm{V}}$ möglicherweise [eines gewaltsamen Todes $]_{\mathrm{Y}}$ - eine steinerne Pfeilspitze steckt in seinem Oberkörper. (St. Galler Tagblatt, 25.09.2001, Ressort: TB-WIS (Abk.); Russ in der Lunge, Flöhe im Kleid)

Auch ASM16 tritt nur bei dem Verb sterben auf, das semantisch mit dem Objektsnomen verwandt ist und durch Adjektive modifiziert wird. Sowohl ASM15 als auch ASM16 unterscheiden sich durch die Ereignisqualität von den anderen Argumentstrukturmustern, sterben ist keine Handlung, sondern ein Prozess.

Die Variante ASM17 der Argumentstrukturmuster, die innere Objekte enthalten, ist formgleich mit ASM14, hat aber eine von diesem deutlich unterschiedene Bedeutung, die so nicht prädiktabel ist. Die Ereignisidentität, die bei ASM14 gegeben ist, kann hier nicht angenommen werden, da der Objektreferent lediglich eines von mehreren möglichen Handlungsmustern bezeichnet, gemäß dem die Handlung ausgeführt wird. Die Variante kommt bei Verben wie tanzen, singen oder spielen vor.

ASM17: einen Tango tanzen

F: $\mathrm{X}_{\text {NPnom }} \mathrm{V} \mathrm{Y}_{\text {NPakk }}$

B: $X$ führt eine Handlung $V$ gemäß dem durch $Y$ bezeichneten Handlungsmuster aus.

ASM17 wird durch Belege wie (18) instanziiert:

(18) $[\text { Wir }]_{\mathrm{X}}[\text { tanzen }]_{\mathrm{V}}[\text { Cha Cha Cha, Rumba und Jive }]_{\mathrm{Y}}$ (lateinamerikanische Tänze), außerdem [Langsamen Walzer, Tango und Quick Step $]_{Y}$ (Standardtänze). Jive und Tango sind meine Lieblingstänze, Quick Step dagegen mag ich nicht so sehr. (Nürnberger Nachrichten, 04.08.2001; Manchmal tun die Füße ganz schön weh - Kerstin Vierkant und Thomas Reichelsdorfer bereiten sich gerade auf Bayerische Meisterschaft vor)

Das Objektsnomen ist in den meisten Fällen mit dem Verb weder morphologisch noch etymologisch verwandt, es besteht allerdings eine enge semantische Verwandtschaft. Nur wenn das Objektsnomen in Form eines Kompositums realisiert wird, kann zumindest beim Grundwort auch etymologische und morphologische Verwandtschaft vorliegen.

In dem Argumentstrukturmuster ASM18 wird ein dem inneren Objekt verwandter Ausdruck in Form einer Präpositionalphrase realisiert: 
ASM18: mit der Laubsäge sägen/mit Meersalz salzen

F: $\mathrm{X}_{\mathrm{NPnom}} \mathrm{V}\left(\mathrm{Z}_{\mathrm{NPakk}}\right) \mathrm{Y}_{\mathrm{PP} m i t}$

B: $X$ führt die Handlung $V$ mit Hilfe von $Y$ in Bezug auf $Z$ aus.

ASM18 wird durch Belege wie in (19) illustriert:

a. Auch das Basteln stand gestern noch einmal hoch im Kurs. [Vincent $]_{\mathrm{X}}(7)[\text { sägte }]_{\mathrm{V}}[\text { mit der Laubsäge }]_{\mathrm{Y}}$ [ein echtes australisches Schwirrholz] $]_{Z}$ (Mannheimer Morgen, 13.10.2001, Ressort: Lokal Lampertheim; Ein letztes Mal erklingen die bunten Didgeridoos)

b. In einem schweren Mörser habe ich mittlerweile schwarze Pfefferkörner und Kümmel zerstoßen; je 1/2 TL für zwei Portionen: über die Kartoffeln streuen und $[\text { diese }]_{\mathrm{Z}}[\text { mit Meersalz }]_{\mathrm{Y}}[\mathrm{sal}-$ zen $]_{\mathrm{V}}$. Den Rest besorgt die Pfanne. (Die Zeit (Online-Ausgabe), 16.01.2003, Nr. 4, Ressort: Leben; Es gibt Bratkartoffeln!)

Die Nominalphrase im Akkusativ bezeichnet in ASM18 das Patiens. Diese Argumentstrukturmustervariante (vgl. auch Levin 1993) tritt entweder bei instrumentinkorporierenden Verben wie sägen, feilen oder hämmern auf oder aber bei Verben, deren Bedeutung sich als „etwas auf/in etwas tun“ paraphrasieren lässt, wie zuckern, salz̨en oder buttern. Die Präpositionalphrase enthält eine Modifikation des normalerweise im Verb inkorporierten Objekts, also in anderer Weise auch eine Argumenterweiterung. Es erfolgt entweder eine Spezifikation des Instruments, mit dessen Hilfe die Handlung ausgeführt wird, oder eine Spezifikation der Substanz, die in oder auf etwas getan wird. Die Nominalphrase in der durch mit eingeleiteten Präpositionalphrase wird hier fast ausnahmslos durch ein Kompositum realisiert, dessen zweiter Bestandteil morphologisch mit dem Verb verwandt ist. Das Nomen der Präpositionalphrase hat die gleiche Referenz wie ein Teil der lexikalischen Paraphrase des Verbs (z.B. für sägen: $x$ zerteilt z mit Hilfe von y \& SÄGE $(y)$; für salzen: $x$ tut $y$ in/auf $z \& \operatorname{SALZ}(y))$. Das ist eine andere Art von Identität als sie für das erste Muster (ASM14) mit inneren Objekten angenommen wurde. Es besteht also sowohl auf der Form- als auch auf der Bedeutungsseite lediglich eine geringe Ähnlichkeit mit ASM14.

Das letzte Argumentstrukturmuster im Bereich der inneren Objekte, ASM19, ist formgleich mit ASM14 und steht darüber und über die weiteren Eigenschaften der Nominalphrase im Akkusativ in einer Ähnlichkeitsbeziehung zu ASM14. 
ASM19: einen Traum träumen

F: $\mathrm{X}_{\mathrm{NPnom}} \mathrm{V} \mathrm{Y}_{\mathrm{NPakk}}$

B: $X$ ist Träger des von $V$ beschriebenen mentalen Zustands oder Ereignisses, und $Y$ bezeichnet den Inhalt oder die Ausprägung des mentalen Zustands.

ASM19 ist durch Beispiele wie (20) belegt:

(20) [Einen Traum $]_{\mathrm{Y}}$ aber [träumte $]_{\mathrm{V}}[\text { Hans Castorp }]_{\mathrm{X}}$ sogar zweimal in dieser Nacht, und zwar beide Male genau in derselben Form, - das letztemal gegen Morgen. (T. Mann: Der Zauberberg. Roman, (1. Buchausg. 1924), In: [Gesammelte Werke in zwölf Bänden mit einem Ergänzungsband], Bd. 3. Frankfurt a.M.: S. Fischer Verlag, 1960)

Die Nominalphrase im Akkusativ ist dabei morphologisch, etymologisch und semantisch mit dem Verb verwandt. Die Verben, die diese Variante von inneren Objekten zulassen, bezeichnen Zustände oder mentale Ereignisse (wie träumen oder glauben) und können sowohl transitiv als auch intransitiv verwendet werden. Auch ASM19 unterscheidet sich durch die Ereignisqualität von den anderen Argumentstrukturmustern. Die Objekts-NP bezeichnet im ASM19 eine komplexe Proposition, nämlich den ganz bestimmten, spezifischen Inhalt des Träumens oder Glaubens, nicht diesen mentalen Zustand oder dieses mentale Ereignis als solches. In einer Variante der betreffenden Verben kann diese komplexe Proposition ja auch unmittelbar als propositionales Objektsatzkomplement realisiert werden (er träumte/glaubte, dass ...). Anders, als das bei normalen Transitivkonstruktionen mit zufälliger formaler Ähnlichkeit zu ASM19 der Fall ist, bezeichnet die Objekts-NP im ASM19 nicht den Gegenstand, auf den die mit dem Verb beschriebene Handlung bezogen ist. ${ }^{14}$

Die Beschreibung der Argumentstrukturmuster ASM14-ASM19 hat gezeigt, dass es sich jeweils um separate Argumentstrukturmuster handelt, die in Relationen von Familienähnlichkeiten zueinander stehen. Keine der Bedeutungen der einzelnen Argumentstrukturmuster kann als die zentrale angesehen werden, auf die alle anderen bezogen sind. ASM14, ASM15, ASM17 und ASM19 haben die gleiche Form, aber unterschiedliche Bedeutungen.

$14 \quad$ Es gibt daneben solche normalen Transitivkonstruktionen, die durch die zufällige morphologische Verwandtschaft des Akkusativobjekts mit dem Verb eine formale Ähnlichkeit mit inneren Objekten aufweisen, wie in dem folgenden Beispiel:

Ich habe keine Ressentiments, ich verzeihe, aber ich vergesse nicht! [Ich $]_{\mathrm{X}}[\text { hasse }]_{\mathrm{V}}$ [den $\mathrm{Haß}]_{\mathrm{Y}},[i c h]_{\mathrm{X}}[\text { liebe }]_{\mathrm{V}}[\text { die Liebe }]_{\mathrm{Y}}$ und ich weiß, wie schwer es ist, ein Held zu sein. (Die Presse, 20.05.1992; Kreativ in vielen Kunstgärten)

Die Nominalphrase im Akkusativ entspricht in solchen Sätzen dem normalen valenzgeforderten Objekt des Verbs, die formale Ähnlichkeit ist nur zufällig. Das wäre auch nach Goldberg ein Fall von Homonymie. Solche formalen Ähnlichkeiten mit inneren Objekten können bei Verben wie lieben, hassen oder träumen auftreten. 
ASM15 und ASM16 haben die gleiche Bedeutung, unterscheiden sich aber in formaler Hinsicht. Die einzelnen Argumentstrukturmuster stehen jeweils in unterschiedlichen, direkten oder vermittelten Ähnlichkeitsbeziehungen zueinander. Anders als bei der Familie der Such-Argumentstrukturmuster kristallisieren sich bei den Argumentstrukturmustern mit inneren Objekten aber keine Subfamilien heraus.

\section{Idiosynkrasien der lexikalischen Füllung von Argumentstrukturmustern}

\subsection{Die Bewegungsvariante von Geräuschverben}

Argumentstrukturmuster sind also als recht feinkörnige, durch Familienähnlichkeiten verknüpfte Entitäten konzipiert und können damit relativ viele Idiosynkrasien des Sprachgebrauchs auffangen. In den Eingangsbeispielen in (1) war allerdings auch von Kookkurrenzen im Argumentstrukturbereich die Rede, die sich auf einer tieferen Abstraktionsebene abspielen. Darum soll es in diesem und den beiden folgenden Abschnitten gehen. In diesem Abschnitt wollen wir am Beispiel der Bewegungslesarten von Geräuschverben demonstrieren, welche Probleme solche Kovorkommen für klassische konstruktionistische Ansätze aufwerfen (vgl. dazu Engelberg 2009).

(21) a. [Die Harleys $]_{\mathrm{X}}[\text { knattern }]_{\mathrm{V}}$ gemütlich [über die märkischen Landstraßen $]_{Y}$, die sportlicheren Maschinen jagen gefährlich um die Kurven. (Berliner Zeitung, 24.06.2000 [S. 35])

b. Weithin hörbar [quietscht $]_{\mathrm{V}}[\mathrm{sie}]_{\mathrm{X}}$ mit atemberaubender Erbarmungslosigkeit [um die Kurve $]_{Y}$, und meine Zähne klappern nun nicht mehr vor Kälte, sondern weil ich sie ob des schmerzenden Geräusches zusammenbeißen muß. (die tageszeitung, 06.03.1990, S. $28 ;$,... wejen de Gleise ..." $)$

c. [Pferdedroschken und Straßenbahnen $]_{\mathrm{X}}[\text { rumpelten }]_{\mathrm{V}}$ [über den einst ruhigen Wittenbergplatz $]_{Y}$ (die tageszeitung, 07.07.1990, S. 39; Inszenierte Rekorde)

Beispiele wie die in (21) werden in konstruktionsgrammatischen Ansätzen im Idealfall so erklärt, dass Verben wie knattern, quietschen oder rumpeln lediglich als einstellige Verben im Lexikon repräsentiert sind, die ihre Bewegungslesart dadurch erhalten, dass sie in eine intransitive Bewegungskonstruktion eingepasst werden, die die PP und die Bewegungslesart lizenziert (Goldberg 2006, S. 100; Welke 2009). Die Erwartung ist dann natürlich, dass sich Geräuschverben in dieser Konstruktion ähnlich wie Bewegungsverben verhalten. Da nun gerade die Direktionalphrase der Indikator für die Bewegungslesart ist, ist zu überprüfen, ob Geräusch- und Bewegungsverben diesbezüglich ein ähnliches Verhalten zeigen. In einer Korpusstudie auf der 


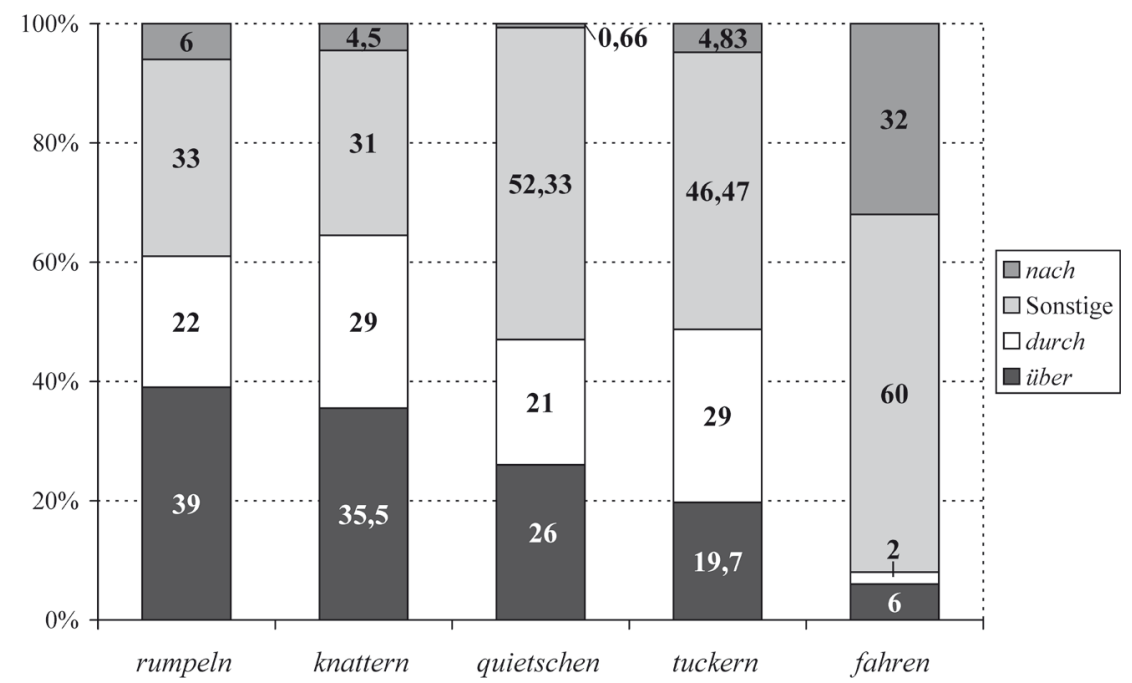

Abb. 1: Verteilung der Präpositionen über, durch und nach bei Geräusch-als-Bewegungsverben und bei fahren

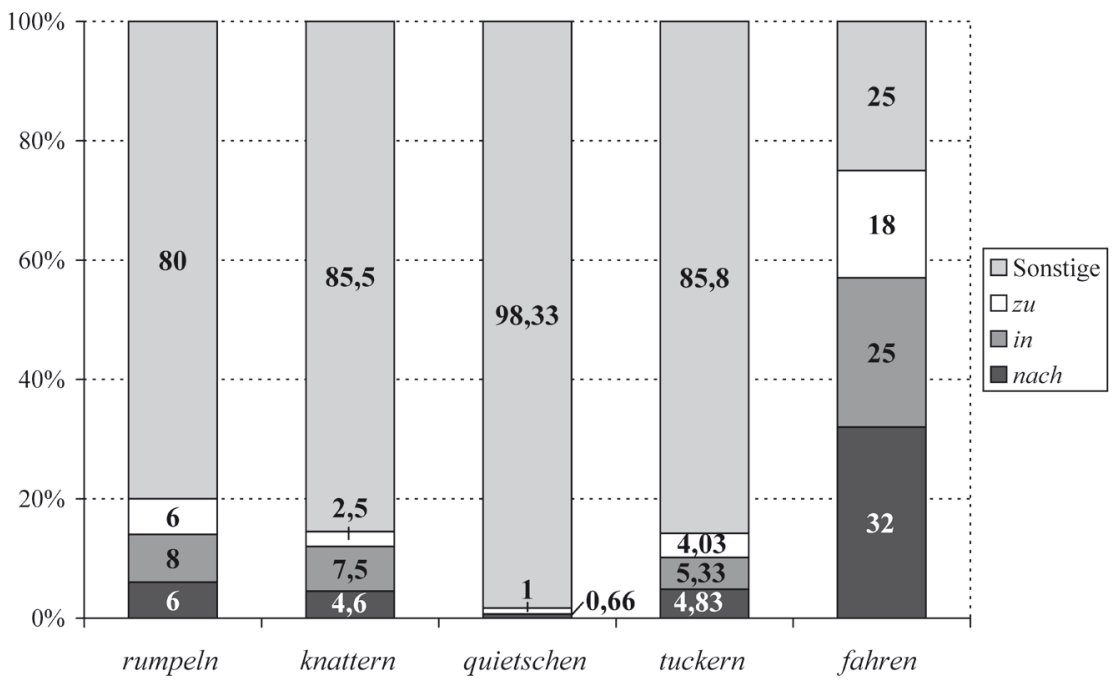

Abb. 2: Verteilung der Präpositionen nach, in und $₹ u$ bei Geräusch-als-Bewegungsverben und bei fabren

Basis von 200 Zufallsbelegen pro Verb zeigte sich allerdings, dass unter den ca. 25 Direktionalausdrücken, die mit einem Geräusch-als-Bewegungsverb kookkurrieren, immer zwei quantitativ herausstechen, nämlich PPs eingeleitet durch über oder durch (siehe auch Engelberg 2009). Sie machen zusam- 
men im Schnitt über 50\% der Belege aus. Die Erwartung, dass auch ein genuines Bewegungsverb wie fahren eine solche Verteilung zeigt, erfüllt sich allerdings nicht (siehe Abbildung 1).

Das Bewegungsverb fahren zeigt ganz andere Präferenzen, nämlich solche für die Präpositionen nach, in und ₹u (siehe Abbildung 2).

Im Gegensatz zu den Geräuschverben, die in ihrer Bewegungslesart wegspezifizierende PPs bevorzugen, zeigt fahren also eine Präferenz für zielspezifizierende PPs. Das lässt etwa Welkes Annahme unplausibel erscheinen, die Bewegungslesart käme ad hoc aufgrund der konzeptuellen Anpassung von Geräuschverben an eine an genuinen Bewegungsverben ausgebildete Bewegungskonstruktion zustande. Die Daten legen vielmehr nahe, von einem eigenen Argumentstrukturmuster für die Bewegungsvariante von Geräuschverben auszugehen, das sich durch besondere Präferenzen für die Realisierung der Direktionalphrase auszeichnet:

ASM20: durchs Dorf knattern

F: $\mathrm{X}_{\mathrm{NPnom}} \mathrm{V} \mathrm{Y}_{\mathrm{PP} / \mathrm{AdvP}[\text { Prä̈erenz für PP durcb und PPiber] }}$

B: $X$ bewegt sich entlang des in $Y$ spezifizierten Pfades und erzeugt dabei das durch $V$ denotierte Geräusch.

In Goldberg/Jackendoff (2004, S. 540 f.) wird für die Bewegungsvariante von Geräuschverben eine eigene Subkonstruktion (,sound-emission path resultative") der Resultativkonstruktion angenommen, allerdings aus einem ganz anderen Grund. Die intransitive Bewegungskonstruktion wird hier durch Ausdrücke wie (22a) exemplifiziert, in der roll die Art und Weise der Bewegung spezifiziert. Bei Geräuschverben wie in (22b) geht es natürlich nicht darum, die Art und Weise der Bewegung auszudrücken, sondern „that the motion causes the sound to be emitted".

a. The ball rolled down the hill.

b. The trolley rumbled down the tunnel.

Über diese semantische Besonderheit rechtfertigen Goldberg/Jackendoff (2004) die Annahme einer eigenen Subkonstruktion..$^{15}$ Dazu ist zu sagen, dass die angenommene semantische Besonderheit eine Erklärung in der bekannten Beschränkung finden könnte, dass zwei Teilereignisse, die über ein einzelnes Verb ausgedrückt werden (also hier Bewegung und Geräuschemission), kausal oder über kausalitätsähnliche Relationen (Kaufmann 1995; Maienborn 1994; Engelberg 2000) verknüpft sein müssen. So kann man nicht sagen er pfeift in den Raum (in der Bedeutung, er kommt pfeifend in den Raum), da Geräusch und Bewegung nicht ursächlich verknüpft sind. Wenn

15 In Goldberg (1995) hätte eine ähnliche Überlegung möglicherweise zur Annahme konstruktioneller Polysemie bei der intransitiven Bewegungskonstruktion geführt. 
man diese Beschränkung so versteht, dass die kausale Verknüpfung nicht unmittelbar sein muss, sondern auch vermittelt sein kann über einen gemeinsamen Verursacher, so ist sie datenadäquater als Goldberg/Jackendoffs (2004) semantische Analyse, die nur für Fälle wie rumpeln und quietschen gilt, nicht aber für Fälle wie knattern und tuckern, bei denen ja nicht die Bewegung das Geräusch hervorruft, sondern sowohl die Bewegung als auch das Geräusch im Normalfall durch die Aktivität des Motors hervorgerufen werden. Die kausale Verknüpfung ist also hier eine andere als die von Goldberg/Jackendoff (2004) angenommene.

Die eigentliche Idiosynkrasie, die zu repräsentieren ist, besteht in der Präferenz der Geräuschverben für die beiden Präpositionen über und durch. Diese ist im Übrigen auch nicht durch semantisch-pragmatische Erfordernisse vollständig zu erklären. Man könnte vermuten, dass gleichzeitige Modusspezifizierung (Geräusch) und Zielspezifizierung dispräferiert sind. Aber selbst wenn das so ist, bleibt die Frage offen, warum unter den vielen wegspezifizierenden Ausdrücken (entlang, vorbei, herum, vorüber, daher, auf, um etc.) ausgerechnet diese beiden bevorzugt werden. Auch die Annahme, dass die räumliche Konfiguration, die die PP beschreibt, ursächlich an der Geräuschentwicklung beteiligt sein muss, führt letztlich nicht zum Ziel. So ist es zwar die räumliche über-Relation zwischen Lastwagen und Straßenbelag, die relevant für die Erzeugung des Geräuschs ist, wenn ein Laster über die Straße rumpelt, aber wenn ein Motorrad durch die Stadt knattert, ist die durch-Relation irrelevant für die Geräuscherzeugung. Die Präpositionspräferenz bleibt also eine Idiosynkrasie des spezifischen Argumentstrukturmusters bzw. der spezifischen Verbklasse der Geräuschverben als Bewegungsverben. Goldberg/Jackendoff (2004) haben demzufolge durchaus recht, wenn sie eine eigene Konstruktion für Geräuschverben als Bewegungsverben ansetzen, aber es gibt schlagkräftigere Argumente als die von ihnen angeführten.

\subsection{Idiosynkrasien bei Resultativprädikaten}

Neben solchen verbklassenspezifischen Restriktionen bei Argumentstrukturmustern sind auch verbspezifische Besonderheiten festzustellen. So arbeiten Stefanowitsch/Gries (2003) heraus, dass Argumentstrukturmuster gewöhnlich eine Präferenz für bestimmte Verben zeigen. Es soll in diesem Abschnitt am Beispiel der subjektreflexiven sowie der transitiven Resultativkonstruktion gezeigt werden, dass darüber hinaus auch die Füllungen weiterer Slots innerhalb eines Argumentstrukturmusters durch Idiosynkrasien geprägt sind.

So bevorzugen beispielsweise Resultativkonstruktionen nicht nur bestimmte Verben, sondern es zeigen sich auch klare Vorlieben bezüglich der Kombination von Verb und Resultativprädikat. Eine korpusbasierte Unter- 
suchung hat ergeben, dass sich im Zusammenspiel von Verb und sekundärem Prädikat innerhalb einer Resultativkonstruktion auffällige Häufigkeitsverteilungen beobachten lassen.

Die subjektreflexive Resultativkonstruktion lässt sich folgendermaßen beschreiben:

ASM21: sich in Rage reden

$\mathrm{F}: \mathrm{X}_{i \text {-NPnom }} \mathrm{VY} \mathrm{Y}_{i \text {-sicb-akk }} \mathrm{Z}_{\mathrm{PP} / \mathrm{AP}}$

B: $X$ führt eine Handlung $V$ aus, die dazu führt, dass $Y(=X)$ in den durch $Z$ beschriebenen Zustand gerät.

ASM21 wird durch Belege wie in (23) illustriert, wobei sich die resultative Bedeutung der Belege nicht aus der Bedeutung der vorkommenden lexikalischen Einheiten vorhersagen lässt; sie ergibt sich erst in der Kombination der verschiedenen Argumente innerhalb des Musters.

(23) a. [Maria Schell $]_{\mathrm{X}-i}[\mathrm{kaufte}]_{\mathrm{V}}[\mathrm{sich}]_{\mathrm{Y}-\mathrm{i}}$ in den Ruin $]_{\mathrm{Z}}$. (Nürnberger Nachrichten, 28.04.2005; Das „Seelchen“ mit dem Tränen-Lächeln wurde nie richtig glücklich)

b. [Viele Jugendliche $]_{\mathrm{X}-i}[\text { essen }]_{\mathrm{V}}[\mathrm{sich}]_{\mathrm{Y}-i}[\mathrm{krank}]_{\mathrm{Z}}$ oder zumindest unglücklich. (Kleine Zeitung, 08.01.1999, Ressort: Lokal; Gesunde Jause gibt es oft nicht einmal in der Pause)

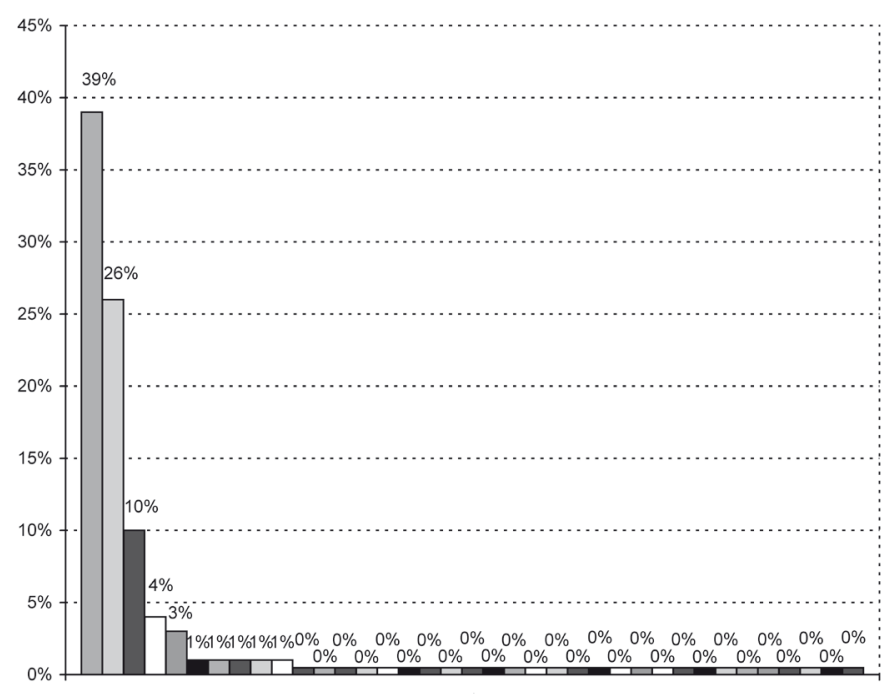

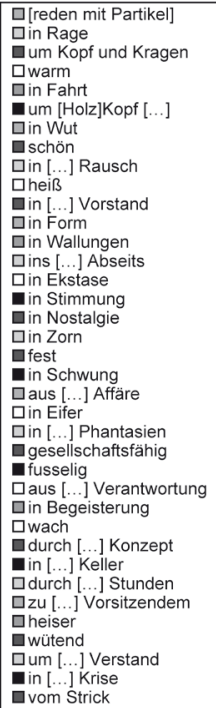

Abb. 3: Häufigkeitsverteilung der Resultativprädikate bei sich reden (Prozentwerte gerundet) 
Es ist nun erkennbar, dass Verben, wenn sie in der subjektreflexiven Resultativkonstruktion vorkommen, Präferenzen haben hinsichtlich des Resultativprädikats, mit dem sie auftreten. Für das Verb reden in der subjektreflexiven Resultativkonstruktion führte eine Korpusuntersuchung zu dem in Abbildung 3 dargestellten Ergebnis.

Diese zu beobachtende auffällige Präferenz des Verbs reden (ohne Verbpartikel) für das sekundäre Prädikat in Rage lässt sich nicht durch die absolute Häufigkeit der Wortform in Rage oder des Lemmas Rage erklären. So kommt Rage außerhalb der Resultativkonstruktion im Vergleich zu anderen Ausdrücken zur Zustandsbeschreibung eines heftigen durch Ärger hervorgerufenen Gefühls deutlich seltener vor: Eine Korpusuntersuchung für die Lemmata Rage, Wut, Zorn, wütend und zornig ergab, dass Rage eindeutig am seltensten im Korpus vertreten ist (Abbildung 4).

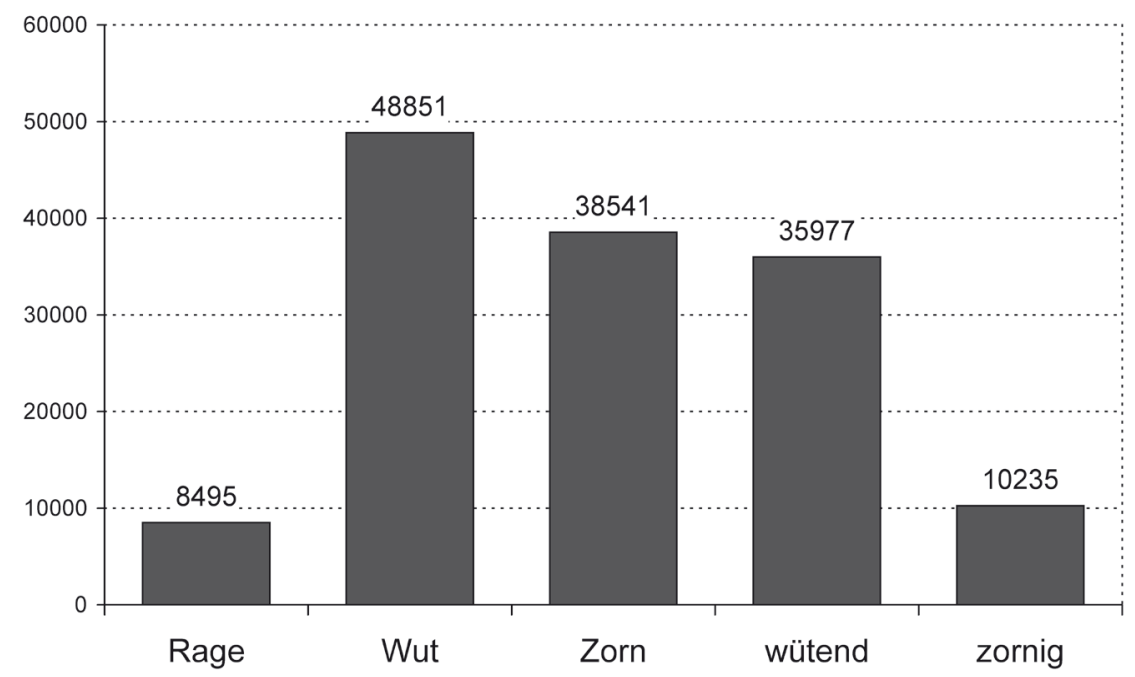

Abb. 4: Vorkommen der Lemmata Rage, Wut, Zorn, wütend und zornig

Auch der feste Ausdruck in Rage kommt verglichen mit den Ausdrücken bzw. Wortformen in Wut, in/im Zorn, wütend und zornig nicht am häufigsten vor (Abbildung 5).

Im Hinblick auf diese quantitativen Ergebnisse ist es umso bemerkenswerter, dass die genannten semantisch ähnlichen Ausdrücke als Resultativprädikate für subjektbezogenes reflexives reden in verschwindend geringer Anzahl vorkommen (Abbildung 6). 


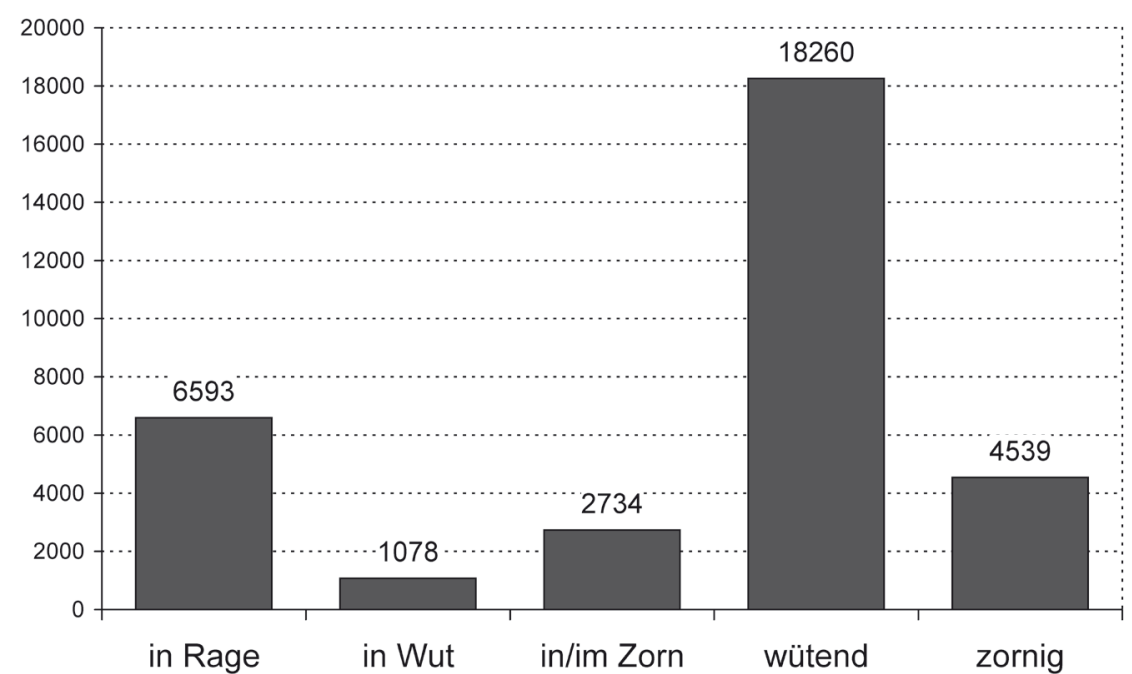

Abb. 5: Vorkommen der Ausdrücke bzw. Wortformen in Rage, in Wut, in Zorn, wütend und zornig

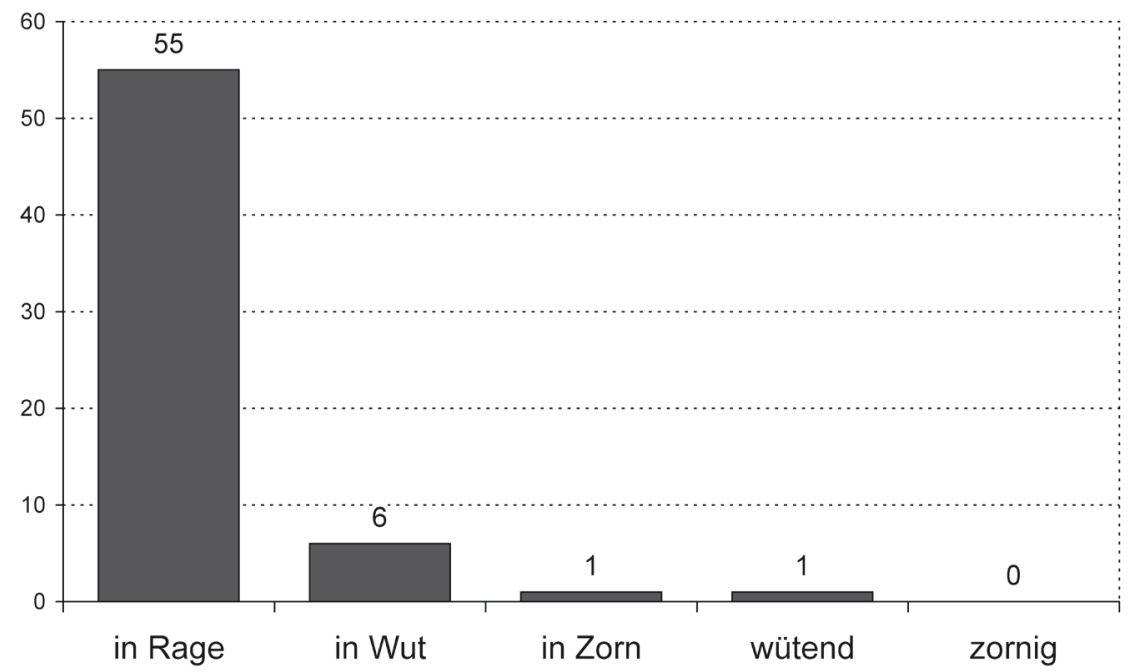

Abb. 6: In Rage, in Wut, in Zorn, wütend und zornig als Resultativprädikate für subjektbezogenes reflexives reden

Weder die Bedeutung von Rage noch allgemeine lexikalische Häufigkeiten oder Weltwissen können erklären, warum subjektreflexives-resultatives reden das Resultativprädikat in Rage so eindeutig präferiert. Gestaltet man nun die Korpusabfrage genau anders herum, untersucht also die Verteilung von Verben aus Sicht des Resultativprädikats, so ergibt sich folgendes Bild: 


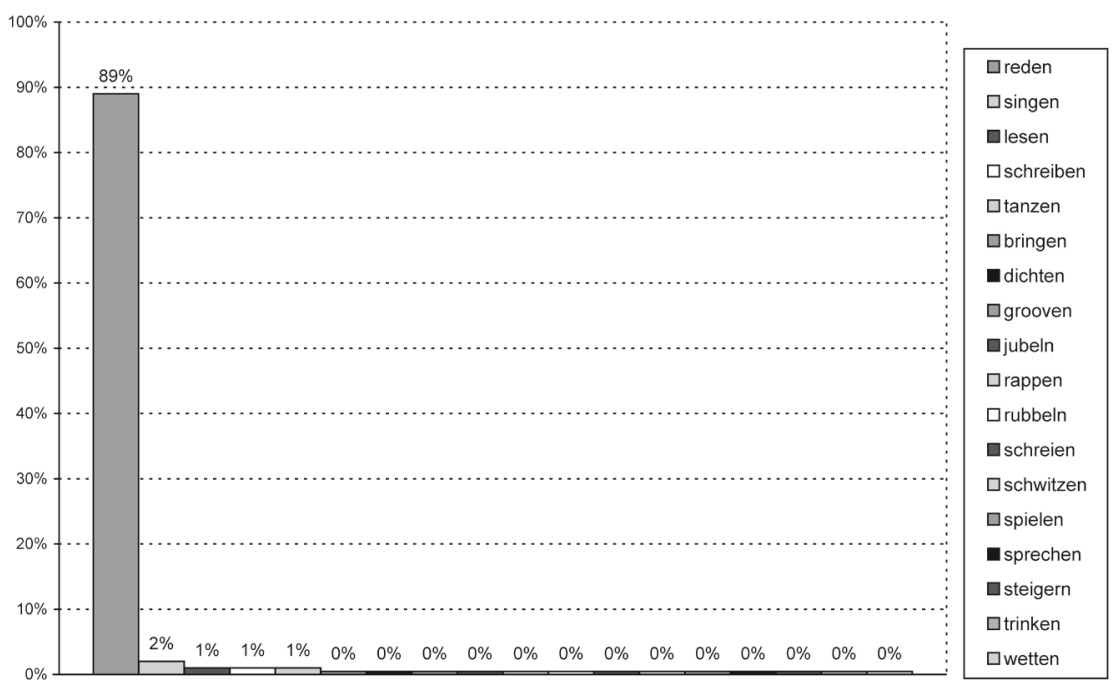

Abb. 7: Verteilung der Verben, die in der subjektreflexiven Resultativkonstruktion mit dem Resultativprädikat in Rage vorkommen (Prozentwerte gerundet)

Hier zeigt sich, dass in Rage mit reden im Rahmen der subjektreflexiven Resultativkonstruktion mit $89 \%$ der Vorkommen deutlich am häufigsten auftritt. Diese starke Präferenz lässt sich wiederum nicht aus generellen Häufigkeiten erklären. So ist reden bei weitem nicht das frequenteste Verb im Korpus. Dass dieses Ergebnis weder auf eine generelle Kollokation von reden und in Rage zurückzuführen ist, noch in Bezug auf eine allgemeine Resultativkonstruktion Gültigkeit hat, zeigt sich in den folgenden Untersuchungen. Zum einen wird bei einer statistischen Kookkurrenzanalyse (Belica 2001-2007) für reden bzw. Rage deutlich, dass beide Lemmata andere Kookkurrenzpartner haben, die (zum Teil wesentlich) häufiger mit ihnen vorkommen. So steht zum Beispiel Rage erst an 47. Stelle der Kookkurrenzpartner von reden, reden als Kookkurrenzpartner für Rage immerhin an fünfter. Zum anderen ergibt sich bereits bei der syntaktisch sehr ähnlichen, in ASM22 dargestellten, nicht-reflexiven transitiven Resultativkonstruktion für das Resultativprädikat in Rage ein abweichendes Bild, wie im Folgenden gezeigt werden soll.

ASM22: jemanden krankenhausreif prügeln

$\mathrm{F}: \mathrm{X}_{\mathrm{NPnom}} \mathrm{V} \mathrm{Y}_{\mathrm{NPakk}} \mathrm{Z}_{\mathrm{PP} / \mathrm{AP}}$

B: $X$ führt eine Handlung $V$ aus, die dazu führt, dass $Y$ in den von $Z$ beschriebenen Zustand gerät. 
ASM22 wird durch Belege wie (24) instanziiert:

(24) Mitten auf dem Golfplatz lauerte [das Känguruh $]_{\mathrm{X}}$ [sic!] dem Buben auf, fiel über ihn her und [prügelte $]_{\mathrm{V}}[\mathrm{ihn}]_{\mathrm{Y}}[\mathrm{krankenhausreif}]_{\mathrm{Z}}$. (Salzburger Nachrichten, 30.10.1998, Ressort: LOKALES; Salzburg im Känguruh-Fieber: Alle suchen den Ausreißer)

Erhebt man nun im Korpus die Daten für das Resultativprädikat in Rage unmittelbar gefolgt von ihn, so erhält man für die vorkommenden Verben folgende Verteilung:

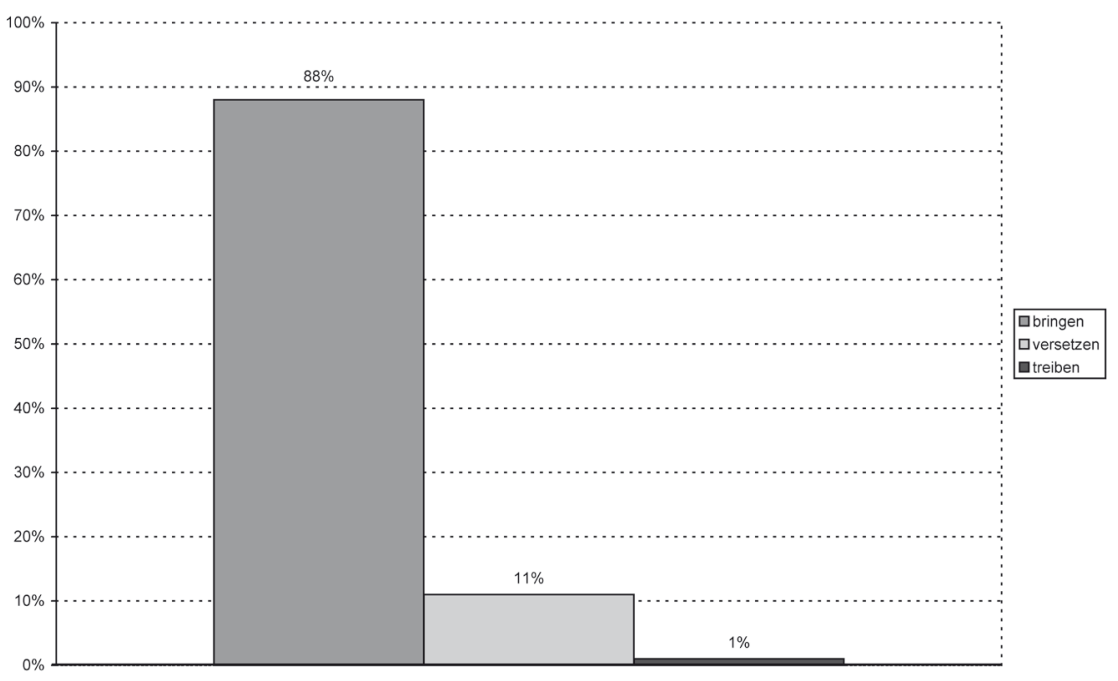

Abb. 8: Verteilung der Verben, die in der transitiven Resultativkonstruktion mit dem Resultativprädikat in Rage vorkommen

In der transitiven Resultativkonstruktion kommt also das Verb bringen am häufigsten mit dem sekundären Prädikat in Rage vor, reden hingegen tritt im Rahmen dieser Untersuchung kein einziges Mal auf. Das Resultativprädikat in Rage zeigt also in den verschiedenen Typen von Resultativkonstruktionen unterschiedliche idiosynkratische Präferenzen für das Verb innerhalb des jeweiligen Argumentstrukturmusters.

\subsection{Idiosynkratische propositionale Muster}

Wir haben in den vergangenen Abschnitten gesehen, dass und wie idiosynkratische Präferenzen für die Füllung von Argumentstellen relativ zu bestimmten Argumentstrukturmustern zustande kommen: Argumentstrukturmuster präferieren bestimmte Verben, Verbklassen präferieren bestimmte Präpositionen in bestimmten Argumentstrukturmustern, Füller von Ar- 
gumentstellen präferieren in bestimmten Argumentstrukturmustern bestimmte Verben. Darüber hinaus ist aber zu beobachten, dass nicht nur Präferenzen für die Füllung einzelner Argumentstellen repräsentiert werden müssen, sondern auch ganze propositionale Muster (vgl. Engelberg 2009). Unter einem propositionalen Muster wollen wir hier die idiosynkratische Kookkurrenz der Argumentstellen eines Verbs in einem Argumentstrukturmuster hinsichtlich der semantischen Klassen verstehen, denen die Ausdrücke angehören, die die Argumentstellen spezifizieren. Das wird im Folgenden an einem Beispiel aus dem Bereich der Bewegungsvariante von Geräuschverben (siehe oben ASM20) erläutert.

Klassifiziert man die Füller der verschiedenen Argumentstellen, die in einer zufälligen Auswahl von 100 Belegen für die Bewegungsvariante von knattern auftreten, so ergibt sich das in Abbildung 9 und 10 dargestellte Bild für die Füllung der Subjekts-NP und die der PP-internen NP. Die Präferenz für bestimmte Präpositionen ist oben in Abbildung 2 ersichtlich.

\begin{tabular}{|l|l|c|}
\hline \multicolumn{1}{|c|}{ Semantische Klasse } & \multicolumn{1}{|c|}{ Lexeme } & Anzahl \\
\hline MOTORRAD & $\begin{array}{l}\text { Simson-Roller, Krafträder, Harley, } \\
\text { Mopeds, Mofa, Wespen etc. }\end{array}$ & 35 \\
\hline LUFTFAHRZEUG & $\begin{array}{l}\text { BH-Hubschrauber, Helikopter, } \\
\text { Fokker, DC-3, Hubschrauber } \text { etc. }\end{array}$ & 25 \\
\hline AUTO & $\begin{array}{l}\text { Ente, Bus, Trabbis, Miniautos, LKW } \\
\text { etc. }\end{array}$ & 17 \\
\hline ANDERE FAHRZEUGE & Schneemobil, Cart etc. & 14 \\
\hline sonstige & Krähen, Akteure, Filmmagazine etc. & 9 \\
\hline
\end{tabular}

Abb. 9: Füllung der Subjekts-NP bei der Bewegunglesart von knattern

\begin{tabular}{|l|l|c|}
\hline \multicolumn{1}{|c|}{ Semantische Klasse } & \multicolumn{1}{|c|}{ Lexeme } & Anzahl \\
\hline $\begin{array}{l}\text { GEOGRAFISCHES } \\
\text { GEBIET }\end{array}$ & $\begin{array}{l}\text { Dörfer, Kramsach, Insel, Wohn- } \\
\text { gebiet, Tal, Wiesen, Oberlausitz, } \\
\text { Hamburg, Wüste } \text { etc. }\end{array}$ & 44 \\
\hline STRASSE & $\begin{array}{l}\text { Rennstrecke, Gleise, Unter den Lin- } \\
\text { den, Ring, Allee etc. }\end{array}$ & 19 \\
\hline GEBÄUDE(TEIL) & Kino, Bühne etc. & 11 \\
\hline sonstige & $\begin{array}{l}\text { Projektoren, Fest, Hand, Lastwagen, } \\
\text { Story, Ruhe etc. }\end{array}$ & 26 \\
\hline
\end{tabular}

Abb. 10: Füllung der PP-internen NP bei der Bewegunglesart von knattern

Nun ist zu beobachten, dass sich die Füller der Subjektstelle, der Präposition und der PP-internen NP präferiert zu propositionalen Mustern verbinden. Die fünf häufigsten Muster aus knattern, einer bestimmten Präposition, 
einer bestimmten semantischen Klasse der Subjekts-NP und einer der PPinternen NP decken, wie in dem innersten Rechteck in Abbildung 11 ersichtlich, bereits 35\% aller Belege ab. Generalisiert man das Muster nach und nach geringfügig, werden bereits $90 \%$ aller Belege erfasst. Das heißt, in $90 \%$ aller Belege für knattern in Bewegungslesart geht es darum, dass ein Fahrzeug auf einem Pfad relativ zu einem Gebiet, einer Straße, einem Gebäude oder einem Gegenstand knattert.

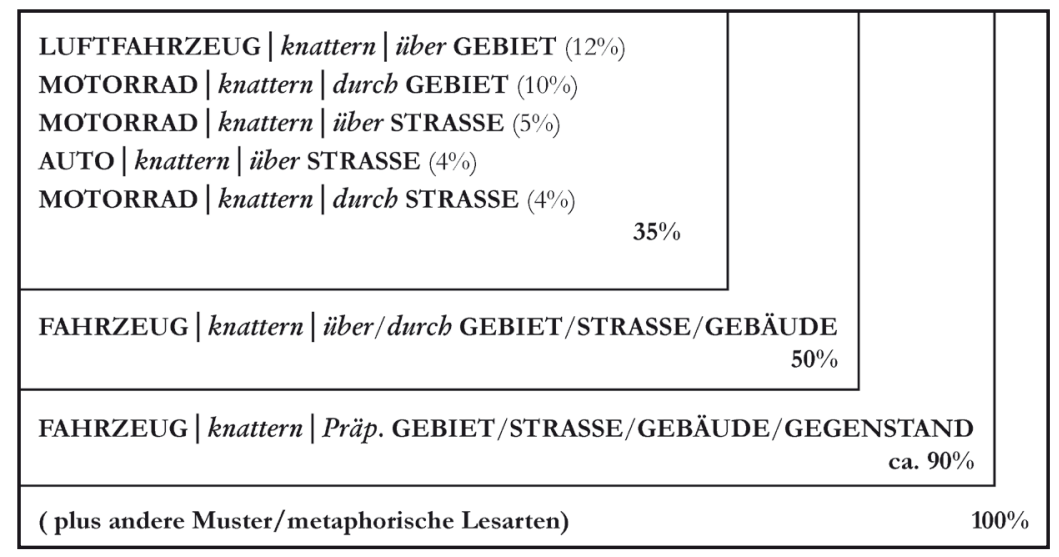

Abb. 11: Propositionale Muster bei knattern in Bewegungslesart

Die restlichen 10\% der Belege sind dagegen weit gestreut und zeigen, dass durchaus auch ganz andere Sachverhalte durch die Bewegungslesart von knattern ausgedrückt werden können:

(25) a. [Salven aus Kalaschnikows $]_{\mathrm{X}}[\text { knatterten }]_{\mathrm{V}}$ [über die Köpfe der außer Rand und Band geratenen Menschenmassen $]_{\mathrm{Y}}[\ldots]$. (Salzburger Nachrichten, 26.11.1991; Belgrad verhöhnt Friedensbemühung von UNO und EG)

b. Gegen Ende der zwölfminütigen Diaschau zur Explosion des Parthenon $[\text { knattern }]_{\mathrm{V}}$ [die Bildmagazine $]_{\mathrm{X}}$ wie verrückt [durch die Projektoren $]_{\mathrm{Y}},[\ldots .$.$] . (die tageszeitung, 16.07.1990, S. 25; Schuß$ in die Pulverkammer)

c. [Knapp 16.500 Bikes $]_{\mathrm{X}}[\text { knatterten }]_{\mathrm{V}} 1994$ [über den Ladentisch $]_{\mathrm{Y}}$, heuer werden es weit mehr als 17.000 sein. (Neue Kronen-Zeitung, 04.04.1995, S. 5)

Die Typen in (25a) und (25b) sind in der Stichprobe jeweils durch zwei ähnliche Beispiele vertreten. Sie konstituieren jeweils eigene propositionale Muster (vgl. Engelberg 2009):

(26) a. SALVE | knattern | ̈̈ber KOPF/PERSON (2\% der Belege)

b. FILM | knattern | durch PROJEKTOR (2\% der Belege) 
Die deutliche Präferenz für Motorräder gegenüber Schüssen in Subjektposition bei der Bewegungsvariante von knattern ist auch über Weltwissen oder thematische Präferenzen nicht zu erklären. Zumindest treten unabhängig von ihrem Vorkommen mit knattern als Bewegungsverb Schüsse in großen Textkorpora nicht seltener auf als Motorräder.

Der Beleg in (25c) wiederum ist ein gutes Indiz für die Existenz des dominierenden 90\%-Musters. Er ist in seiner stilistischen Funktion ohne die Vertrautheit mit Sätzen dieses Musters wohl kaum zu verstehen. Nur auf dieser Basis kann die Amalgamierung von ,über [Straßen] knattern“ und „über den Ladentisch [gehen]" ihre Funktion entfalten.

Wir konstatieren also, dass offenbar ganze propositionale Muster als Spezifizierung bestimmter Argumentstrukturmuster kognitiv präsent sind. Das steht auch in Einklang mit neueren psycholinguistischen Tests, die zeigen, dass Sprecher offenbar ganze propositionale Einheiten aus Gesprächen über längere Zeit memorieren (Gurevich/Johnson/Goldberg 2010).

Die in den Abschnitten 3.1 bis 3.3 beschriebenen Phänomene stellen alle verschiedenen Arten von idiosynkratischen lexikalischen Kookkurrenzen innerhalb einzelner Argumentstrukturmuster dar, die offenbar Teil unseres sprachlichen Wissens sind. Als Spezifizierungen eines Argumentstrukturmusters berühren sie die generellen Eigenschaften eines solchen Musters nicht. Sie lassen sich über implikative Beziehungen oder Vererbungshierarchien mit der allgemeinen Repräsentation des Musters in Beziehung setzen.

\section{Theoretische Einordnung der Befunde}

Ziel dieses Artikels war es zu zeigen, wie Argumentstrukturmuster identifiziert werden können, wie die Beziehungen, in denen sie zueinander stehen, erfasst werden können, und welche Arten idiosynkratischer lexikalischer Kookkurrenzen innerhalb solcher Muster repräsentiert werden müssen. Das Unterfangen war im Wesentlichen um deskriptive Adäquatheit bemüht, verbunden mit angemessenen Generalisierungen. Es war nicht beabsichtigt zu zeigen, wie die beschriebenen Restriktionen und Regelhaftigkeiten solcher Muster sprachtheoretisch angemessen erfasst werden können. In dem $\mathrm{Zu}-$ sammenhang ist zu sagen, dass natürlich auch nicht behauptet werden sollte, dass alle deskriptiven Elemente in den dargestellten Mustern idiosynkratischer Natur sind. Die jeweils repräsentierte Nominalphrase im Nominativ ist ja keine Idiosynkrasie eines einzelnen Argumentstrukturmusters. Jedes der angeführten Muster enthält allerdings Idiosynkrasien, von denen wir annehmen, dass sie nicht aus anderen Festlegungen der Grammatik vollständig herleitbar sind.

In weiten Bereichen ist der Auffassung Jacobs' (2009) zuzustimmen, dass Kookkurrenzen im Bereich von Argumentstrukturen deskriptiv adä- 
quat über konstruktionsbasierte oder valenzbasierte projektionistische Modelle beschrieben werden können. $\mathrm{Ob}$ also die Argumentstrukturmuster als Konstruktionen beschrieben werden, die bestimmte Verben selegieren, oder aber als lexikalische Redundanzregeln, die über argumentstrukturell voll spezifizierten lexikalischen Einträgen operieren, ist unter dem Aspekt deskriptiver Adäquatheit für die meisten der betrachteten Phänomene unerheblich. Es hat sich aber auch gezeigt, dass manche Präferenzen für die Füllung von Argumentstellen sich nicht an valenz- und damit weitgehend kopforientierte Beschreibungen von Umgebungsrestriktionen anbinden lassen. Wie etwa im Fall der Idiosynkrasien bei Resultativkonstruktionen zu sehen war, gehen Selektionspräferenzen in bestimmten Argumentstrukturmustern nicht unbedingt vom Kopf des Musters aus. Auch die Indizien für das Vorliegen ganzer propositionaler Muster kommen valenzbasierten Vorstellungen, dass einzelne Argumente unabhängig voneinander durch das Verb semantisch beschränkt werden, nicht unbedingt nahe.

In noch stärkerem Maße widersprechen die Daten allerdings solchen konstruktionsbasierten Theorien, die annehmen, dass Verben bestimmter Bedeutungsklassen (z.B. Geräuschverben) in quasi fremde Argumentstrukturmuster (z.B. eine intransitive Bewegungskonstruktion) eingesetzt werden können und im Zuge einer konzeptuellen Anpassung dort ad hoc im Sprachverarbeitungsprozess verfügbar gemacht werden. Die beobachteten Idiosynkrasien sprechen hier eindeutig gegen solche pragmatisch gesteuerten konstruktionsgrammatischen Ansätze. Wenn eine konstruktionsgrammatische Modellierung dieser Phänomene angestrebt ist, dann muss sie von pragmatisch gesteuerten Ad-hoc-Prozessen weitgehend absehen, und stattdessen von einer Vielzahl äußerst feinkörnig gestalteter Konstruktionen als Bestandteil unseres gespeicherten syntaktisch-semantischen Wissens ausgehen.

Unsere Daten legen insbesondere nahe, dass der Konstruktionsgedanke vor allem auf einer sehr ausdrucksnahen, konkreten Ebene greift. Die idiosynkratischen Präferenzen für bestimmte Argumentstellen in bestimmten Argumentstrukturmustern suggerieren, dass solche Idiosynkrasien in Form konstruktionsähnlicher Gebrauchsmuster kognitiv präsent sind. Auf der abstrakteren Ebene scheint uns die Frage nach der sprachtheoretischen Angemessenheit von konstruktionsbasierten versus regelgesteuerten valenzbasierten Modellen noch nicht beantwortet. Es ist zumindest deutlich geworden, dass die Varianz bei Argumentstrukturmustern erheblich größer ist, als dies im Allgemeinen angenommen wird, und dass jedes dieser hier recht granular erfassten Muster Idiosynkrasien in Form und/oder Bedeutung einerseits und bezüglich der Einsetzbarkeit von Verben andererseits aufweist. Zumindest auf einer deskriptiven Ebene konstituieren solche Muster eher ein Netz von Familienähnlichkeiten als eine rein taxonomische oder polysemiebasierte Struktur. 


\section{Anhang}

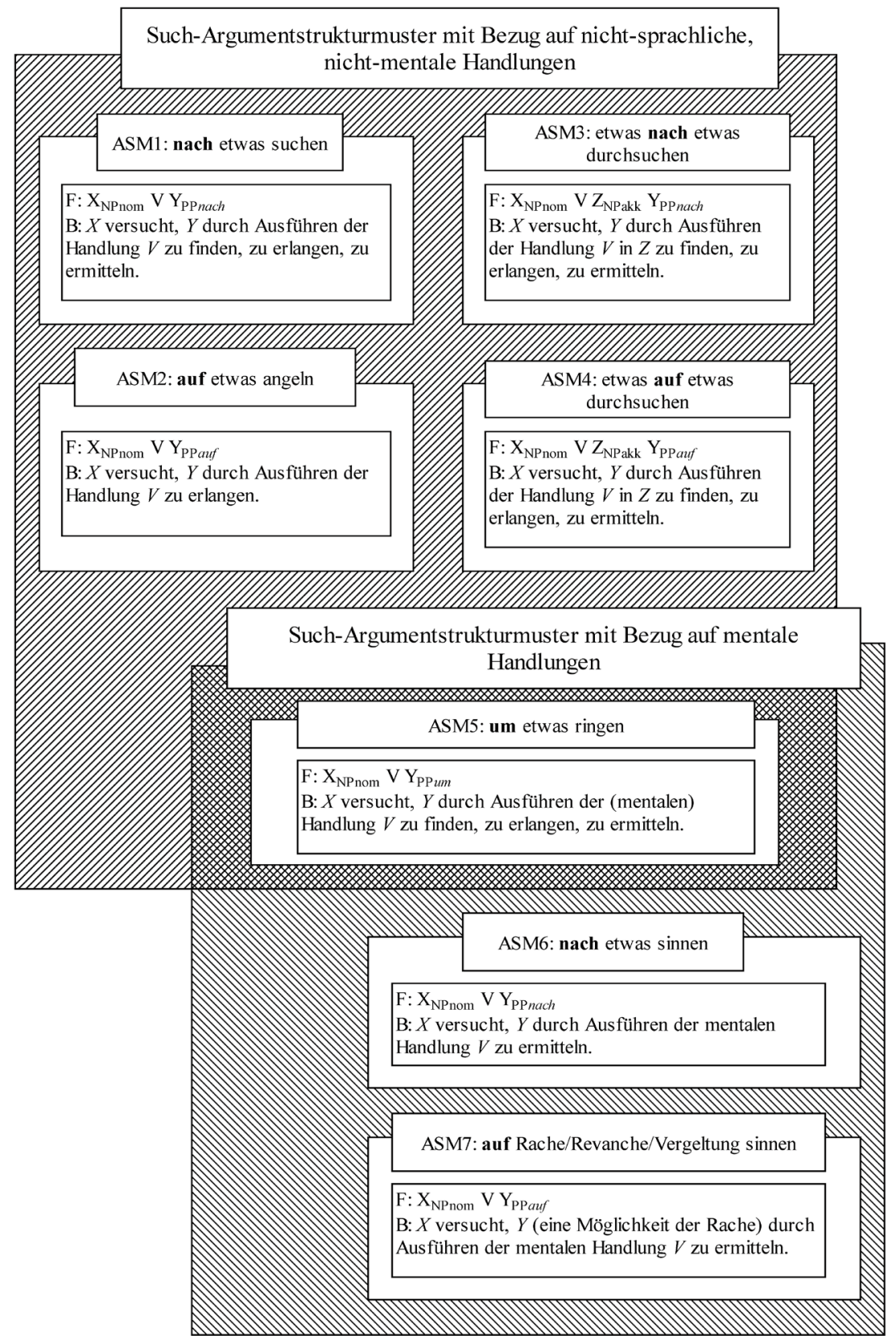



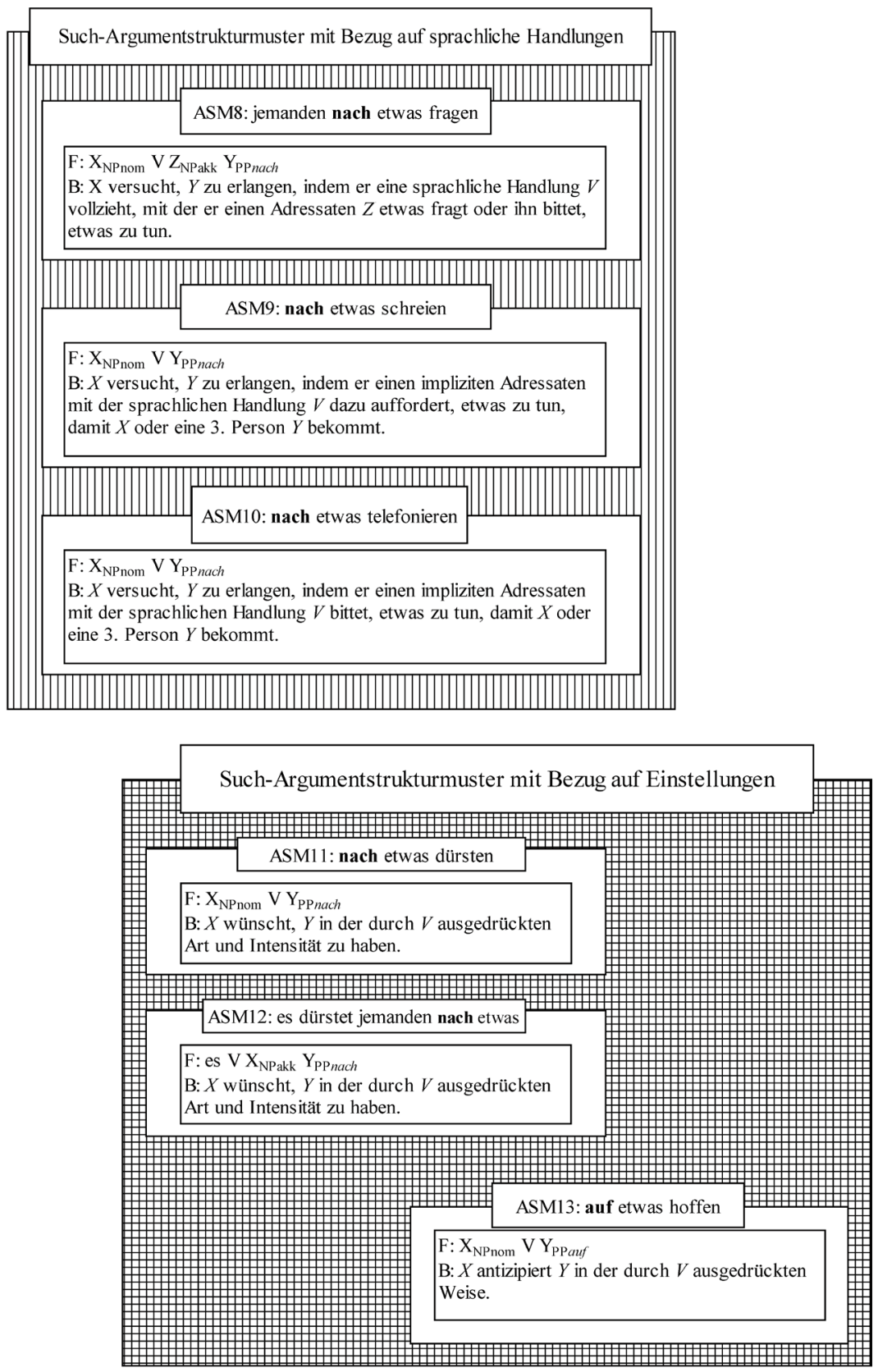

Abb. 12: Die Familie der Such-Argumentstrukturmuster 


\begin{tabular}{|c|c|c|c|c|c|c|c|c|c|}
\hline \multirow[t]{3}{*}{ ASM } & \multicolumn{3}{|c|}{ Semantische Ähnlichkeiten } & \multicolumn{5}{|c|}{ Formale Ähnlichkeiten } & \multirow{3}{*}{$\begin{array}{c}\text { Ausmaß } \\
\text { der } \\
\text { Ähn- } \\
\text { lichkeit }\end{array}$} \\
\hline & \multirow{2}{*}{$\begin{array}{l}\text { Vorkom- } \\
\text { men in } \\
\text { gleicher } \\
\text { Subfamilie }\end{array}$} & \multicolumn{2}{|c|}{$\begin{array}{c}\text { Übereinstimmung } \\
\text { Anzahl der } \\
\text { Argumente }\end{array}$} & \multicolumn{3}{|c|}{$\begin{array}{l}\text { Übereinstimmung } \\
\text { Präposition }\end{array}$} & \multicolumn{2}{|c|}{$\begin{array}{c}\text { Übereinstimmung } \\
\text { phrasaler } \\
\text { Kategorien } \\
\end{array}$} & \\
\hline & & 2 Arg. & 3 Arg. & PPnach & PPauf & PРиm & NPnom & NPakk & \\
\hline ASM1/ASM2 & * & * & & & & & * & & 3 \\
\hline ASM1/ASM3 & $*$ & & & $*$ & & & $*$ & & 3 \\
\hline ASM1/ASM4 & * & & & & & & * & & 2 \\
\hline ASM1/ASM5 & & $*$ & & & & & $*$ & & 2 \\
\hline ASM1/ASM6 & & $*$ & & $*$ & & & $*$ & & 3 \\
\hline ASM1/ASM7 & & * & & & & & $*$ & & 2 \\
\hline ASM1/ASM8 & & & & $*$ & & & $*$ & & 2 \\
\hline ASM1/ASM9 & & * & & $*$ & & & $*$ & & 3 \\
\hline ASM1/ASM10 & & * & & * & & & * & & 3 \\
\hline ASM1/ASM11 & & $*$ & & $*$ & & & * & & 3 \\
\hline ASM1/ASM12 & & $*$ & & $*$ & & & & & 2 \\
\hline ASM1/ASM13 & & $*$ & & & & & $*$ & & 2 \\
\hline ASM2/ASM3 & $*$ & & & & & & $*$ & & 2 \\
\hline ASM2/ASM4 & $*$ & & & & $*$ & & $*$ & & 3 \\
\hline ASM2/ASM5 & & * & & & & & $*$ & & 2 \\
\hline ASM2/ASM6 & & * & & & & & $*$ & & 2 \\
\hline ASM2/ASM7 & & $*$ & & & $*$ & & $*$ & & 3 \\
\hline ASM2/ASM8 & & & & & & & * & & 1 \\
\hline ASM2/ASM9 & & $*$ & & & & & $*$ & & 2 \\
\hline ASM2/ASM10 & & $*$ & & & & & $*$ & & 2 \\
\hline ASM2/ASM11 & & * & & & & & $*$ & & 2 \\
\hline ASM2/ASM12 & & $*$ & & & & & & & 1 \\
\hline ASM2/ASM13 & & $*$ & & & $*$ & & $*$ & & 3 \\
\hline ASM3/ASM4 & * & * & & & & & $*$ & $*$ & 4 \\
\hline ASM3/ASM5 & & & & & & & * & & 1 \\
\hline ASM3/ASM6 & & & & $*$ & & & $*$ & & 2 \\
\hline ASM3/ASM7 & & & & & & & $*$ & & 1 \\
\hline ASM3/ASM8 & & & * & $*$ & & & * & $*$ & 4 \\
\hline ASM3/ASM9 & & & & $*$ & & & $*$ & & 2 \\
\hline ASM3/ASM10 & & & & $*$ & & & $*$ & & 2 \\
\hline ASM3/ASM11 & & & & $*$ & & & $*$ & & 2 \\
\hline ASM3/ASM12 & & & & $*$ & & & & $*$ & 2 \\
\hline ASM3/ASM13 & & & & & & & $*$ & & 1 \\
\hline ASM4/ASM5 & & & & & & & $*$ & & 1 \\
\hline ASM4/ASM6 & & & & & & & $*$ & & 1 \\
\hline ASM4/ASM7 & & & & & & & $*$ & & 1 \\
\hline ASM4/ASM8 & & & $*$ & & & & $*$ & $*$ & 3 \\
\hline ASM4/ASM9 & & & & & & & $*$ & & 1 \\
\hline ASM4/ASM10 & & & & & & & $*$ & & 1 \\
\hline ASM4/ASM11 & & & & & & & $*$ & & 1 \\
\hline
\end{tabular}




\begin{tabular}{|c|c|c|c|c|c|c|c|c|c|}
\hline \multirow{3}{*}{ ASM } & \multicolumn{3}{|c|}{ Semantische Ähnlichkeiten } & \multicolumn{5}{|c|}{ Formale Ähnlichkeiten } & \multirow{3}{*}{$\begin{array}{c}\text { Ausmaß } \\
\text { der } \\
\text { Ähn- } \\
\text { lichkeit }\end{array}$} \\
\hline & \multirow{2}{*}{\begin{tabular}{|c|} 
Vorkom- \\
men in \\
gleicher \\
Subfamilie
\end{tabular}} & \multicolumn{2}{|c|}{$\begin{array}{c}\text { Übereinstimmung } \\
\text { Anzahl der } \\
\text { Argumente }\end{array}$} & \multicolumn{3}{|c|}{$\begin{array}{l}\text { Übereinstimmung } \\
\text { Präposition }\end{array}$} & \multicolumn{2}{|c|}{$\begin{array}{c}\text { Übereinstimmung } \\
\text { phrasaler } \\
\text { Kategorien } \\
\end{array}$} & \\
\hline & & 2 Arg. & 3 Arg. & PPnach & PPauf & PРum & NPnom & NPakk & \\
\hline ASM4/ASM12 & & & & & & & & * & 1 \\
\hline ASM4/ASM13 & & & & & * & & * & & 2 \\
\hline ASM5/ASM6 & * & * & & & & & * & & 3 \\
\hline ASM5/ASM7 & $*$ & $*$ & & & & & * & & 3 \\
\hline ASM5/ASM8 & & & & & & & * & & 1 \\
\hline ASM5/ASM9 & & $*$ & & & & & * & & 2 \\
\hline ASM5/ASM10 & & * & & & & & $*$ & & 2 \\
\hline ASM5/ASM11 & & $*$ & & & & & $*$ & & 2 \\
\hline ASM5/ASM12 & & * & & & & & & & 1 \\
\hline ASM5/ASM13 & & $*$ & & & & & * & & 2 \\
\hline ASM6/ASM7 & * & * & & & & & $*$ & & 3 \\
\hline ASM6/ASM8 & & 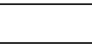 & & $*$ & & & $*$ & & 2 \\
\hline ASM6/ASM9 & & $*$ & & $*$ & & & $*$ & & 3 \\
\hline ASM6/ASM10 & & * & & $*$ & & & * & & 3 \\
\hline ASM6/ASM11 & & * & & $*$ & & & * & & 3 \\
\hline ASM6/ASM12 & & $*$ & & $*$ & & & - & & 2 \\
\hline ASM6/ASM13 & & $*$ & & & & & $*$ & & 2 \\
\hline ASM7/ASM8 & & & & & & & * & & 1 \\
\hline ASM7/ASM9 & & $*$ & & & & & $*$ & & 2 \\
\hline ASM7/ASM10 & & $*$ & & 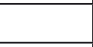 & & & $*$ & & 2 \\
\hline ASM7/ASM11 & & $*$ & & & & & $*$ & & 2 \\
\hline ASM7/ASM12 & & * & & & & & & & 1 \\
\hline ASM7/ASM13 & & $*$ & & & * & & $*$ & & 3 \\
\hline ASM8/ASM9 & $*$ & - & & $*$ & & & $*$ & & 3 \\
\hline ASM8/ASM10 & $*$ & 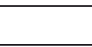 & & $*$ & & & $*$ & & 3 \\
\hline ASM8/ASM11 & & & & $*$ & & & $*$ & & 2 \\
\hline ASM8/ASM12 & & & & * & & & & * & 2 \\
\hline ASM8/ASM13 & & & & & & & $*$ & & 1 \\
\hline ASM9/ASM10 & $*$ & $*$ & & $*$ & & & $*$ & & 4 \\
\hline ASM9/ASM11 & & $*$ & & $*$ & & & $*$ & & 3 \\
\hline ASM9/ASM12 & & * & & $*$ & & & & & 2 \\
\hline ASM9/ASM13 & & $*$ & & 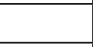 & & & $*$ & & 2 \\
\hline ASM10/ASM11 & & $*$ & & $*$ & & & $*$ & & 3 \\
\hline ASM10/ASM12 & & $*$ & & $*$ & & & & & 2 \\
\hline ASM10/ASM13 & & $*$ & & & & & $*$ & & 2 \\
\hline ASM11/ASM12 & $*$ & $*$ & & $*$ & & & & & 3 \\
\hline ASM11/ASM13 & $*$ & $*$ & & & & & $*$ & & 3 \\
\hline ASM12/ASM13 & $*$ & $*$ & & & & & & & 2 \\
\hline
\end{tabular}

Tab. 1: Semantische und formale Ähnlichkeiten von Such-Argumentstrukturmustern 


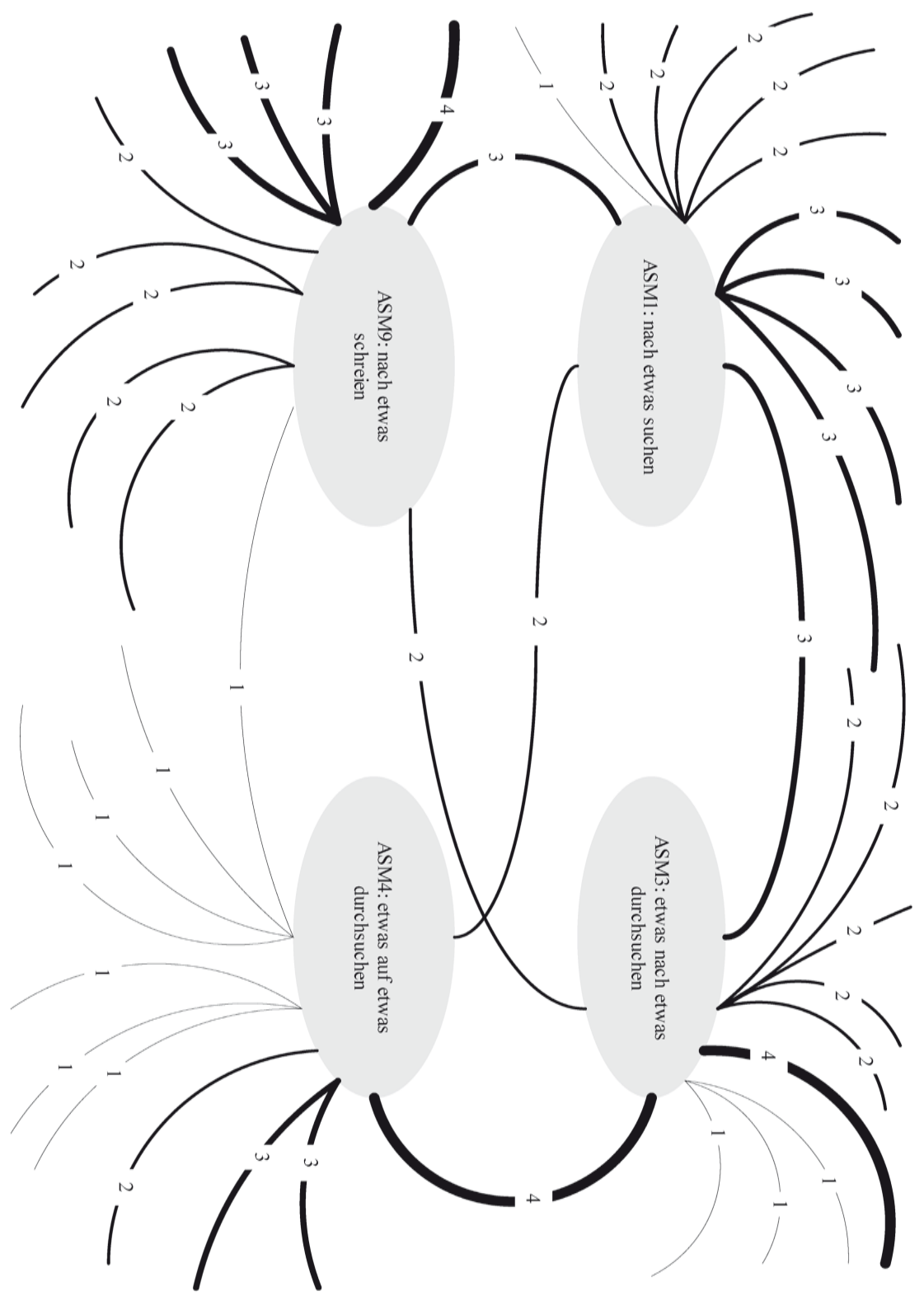

Abb. 13: Grad der Ähnlichkeit von Such-Argumentstrukturmustern 


\section{Literatur}

Baron, Naomi (1971): On defining cognate object. In: Glossa 0017-1271, S. 71-98.

Belica, Cyril (2001-2007): Kookkurrenzdatenbank CCDB - V3.2. Eine korpuslinguistische Denk- und Experimentierplattform. Institut für Deutsche Sprache, Mannheim.

Engelberg, Stefan (2000): Verben, Ereignisse und das Lexikon. (= Linguistische Arbeiten 414). Tübingen.

Engelberg, Stefan (2007): Konstruktionelle Varianten zwischen Wörterbuch und Grammatik. In: Germanistische Mitteilungen 66, S. 11-27.

Engelberg, Stefan (2009): Blätter knistern über den Beton. Zwischenbericht aus einer korpuslinguistischen Studie zur Bewegungsinterpretation bei Geräuschverben. In: Winkler (Hg.), S. 75-97. Internet: www.ids-mannheim.de/pub/laufend/opal/privat/pdf/opal09-4 _engelberg.pdf (Stand: 08.11.2010).

Goldberg, Adele E. (1995): Constructions: a Construction Grammar approach to argument structure. Chicago.

Goldberg, Adele E. (1997): The relationships between verbs and constructions. In: Verspoor, Marjolijn H./Kee Dong, Lee/Sweetser, Eve (Hg.): Lexical and syntactical constructions and the construction of meaning. (= Current Issues in Linguistic Theory 150). Amsterdam/Philadelphia, S. 383-398.

Goldberg, Adele E. (2006): Constructions at work. The nature of generalization in language. Oxford.

Goldberg, Adele E. (2009): The nature of generalization in language. In: Cognitive Linguistics 20, S. 93-127.

Goldberg, Adele E./Jackendoff, Ray (2004): The English resultative as a family of constructions. In: Language 80, 3, S. 532-568.

Gurevich, Olga/Johnson, Matthew A./Goldberg, Adele E. (2010): Incidental verbatim memory for language. In: Language and Cognition 21, S. 45-78.

Handwerker, Brigitte (2008): Lernbasis Lexikon. - Das Verb und die Lizenz zu konstruieren. In: Fandrych, Christian/Thonhauser, Ingo (Hg.): Fertigkeiten und Kompetenzen - separiert oder integriert? Zur Neubewertung der Fertigkeiten und Kompetenzen im Fremdsprachenunterricht. Wien, S. 35-54.

Harley, Heidi/Noyer, Rolf (2000): Formal versus encyclopedic properties of vocabulary: evidence from nominalizations. In: Peeters, Bert (Hg.): The lexicon-encyclopedia interface. (= Current Research in the Semantics-Pragmatics Interface 5). Amsterdam, S. 349-375.

Höche, Silke (2009): Cognate object constructions in English: a cognitive-linguistic account. (= Language in Performance 41). Tübingen.

Jacobs, Joachim (1992): Bewegung als Valenzvererbung, Teil I. In: Linguistische Berichte 138 , S. 85-122.

Jacobs, Joachim (1994): Kontra Valenz. Trier. 
Jacobs, Joachim (2008): Wozu Konstruktionen? In: Linguistische Berichte 213, S. 3-44.

Jacobs, Joachim (2009): Valenzbindung oder Konstruktionsbindung? Eine Grundfrage der Grammatiktheorie. In: Zeitschrift für germanistische Linguistik 37, 3, S. 490513.

Kaufmann, Ingrid (1995): Konzeptuelle Grundlagen semantischer Dekompositionsstrukturen. Die Kombinatorik lokaler Verben und prädikativer Komplemente. (= Linguistische Arbeiten 335). Tübingen.

Levin, Beth (1993): English verb classes and alternations. A preliminary investigation. Chicago.

Maienborn, Claudia (1994): Kompakte Strukturen: Direktionale Präpositionalphrasen und nicht-lokale Verben. In: Felix, Sascha W./Habel, Christopher/Rickheit, Gert (Hg.): Kognitive Linguistik. Repräsentation und Prozesse. Opladen, S. 229-249.

Matsumoto, Masumi (1996): The syntax and semantics of the cognate object construction. In: English Linguistics (Journal of the English Linguistic Society of Japan) 13, S. $190-220$.

Müller, Stefan (2006): Resultativkonstruktionen, Partikelverben und syntaktische vs. lexikonbasierte Konstruktionen. In: Fischer, Kerstin/Stefanowitsch, Anatol (Hg.): Konstruktionsgrammatik. Von der Anwendung zur Theorie. (= Stauffenburg Linguistik 40). Tübingen, S. 177-202.

Proost, Kristel (2009): Warum man nach Schnäppchen jagen, aber nicht nach Klamotten bummeln kann. Die nach-Konstruktion zwischen Lexikon und Grammatik. In: Winkler (Hg.), S. 10-41. Internet: www.ids-mannheim.de/pub/laufend/opal/privat/ pdf/opal09-4_proost.pdf (Stand: 08.11.2010).

Stefanowitsch, Anatol/Gries, Stefan T. (2003): Collostructions: Investigating the interaction of words and constructions. In: International Journal of Corpus Linguistics 8, 2, S. 209-243.

Welke, Klaus (2009): Valenz und Konstruktionsgrammatik. In: Zeitschrift für Germanistische Linguistik 37, S. 81-124.

Winkler, Edeltraud (2009): Anna lächelte ibr freundliches Lächeln. Syntaktischer und semantischer Status von inneren Objekten im Deutschen. In: Winkler (Hg.), S. 126145. Internet: www.ids-mannheim.de/pub/laufend/opal/privat/pdf/opal09-4_winkler.pdf (Stand: 08.11.2010).

Winkler, Edeltraud (Hg.) (2009): Konstruktionelle Varianz bei Verben. (= OPAL - Online publizierte Arbeiten zur Linguistik Sonderheft 4/2009). Mannheim. Internet: www.ids-mannheim.de/pub/laufend/opal/privat/opal09-4.html (Stand: 08.11.2010).

Wittgenstein, Ludwig (2001): Philosophische Untersuchungen. Kritisch-genetische Edition. Hrsg. v. Joachim Schulte. Frankfurt a.M.

Wunderlich, Dieter (2006): Towards a structural typology of verb classes. In: Wunderlich, Dieter (Hg.): Advances in the theory of the lexicon. (= Interface Explorations 13). Berlin/New York, S. 57-166. 